\title{
Catalytic Asymmetric Total Synthesis of (+)-Yohimbine
}

\author{
Dustin J. Mergott, Stephan J. Zuend, and Eric N. Jacobsen* \\ Department of Chemistry and Chemical Biology, Harvard University, Cambridge, MA, 02138 \\ email: jacobsen@chemistry.harvard.edu
}

SUPPORTING INFORMATION

PART 1: EXPERIMENTAL STUDIES pp. S-1-S-21

PART 2: COMPUTATIONAL STUDIES pp. S-22-S-31 
Experimental Methods: All reaction solvents were purified before use. Tetrahydrofuran and ether were purified by distillation from sodium benzophenone ketyl. Dichloromethane, acetonitrile, diisopropylamine, diisopropylethylamine, and dimethylsulfoxide were purified by distillation from calcium hydride. Methanol was purified by distillation from magnesium turnings. Acetyl chloride, 2,6-lutidine, borane-ammonia complex, n-butyllithium, sodium cyanoborohydride, benzoic acid, potassium bis(trimethylsilylamide), sulfurtrioxide-pyridine complex, methyl(triphenylphosphoranylidene) acetate, trifluoroethanol, scandium triflate, cesium carbonate, and $10 \% \mathrm{Pd} / \mathrm{C}$ were purchased from Aldrich and used as received. All reactions sensitive to moisture or oxygen were conducted under a nitrogen or argon atmosphere using flame-dried or oven-dried $\left(150{ }^{\circ} \mathrm{C}\right.$, overnight) glassware. House nitrogen was passed through a column of anhydrous calcium sulfate prior to use. Removal of solvents was accomplished on a rotary evaporator at reduced pressure.

Physical Properties and Spectroscopic Measurements: Proton nuclear magnetic resonance $\left({ }^{1} \mathrm{H}\right.$ NMR) spectra and carbon-13 $\left({ }^{13} \mathrm{C}\right)$ NMR spectra were recorded on a Varian Mercury-400 spectrometer at $400 \mathrm{MHz}$ or on a Varian Inova-500 spectrometer at $500 \mathrm{MHz}$. The proton signal of residual, non-deuterated solvent $\left(\delta 7.26 \mathrm{ppm}\right.$ for $\left.\mathrm{CHCl}_{3}\right)$ was used as an internal reference for ${ }^{1} \mathrm{H}$ spectra measured in these solvents. For ${ }^{13} \mathrm{C}$ spectra, chemical shifts are reported relative to the $\delta 77.0$ ppm resonance of $\mathrm{CDCl}_{3}$. Coupling constants are reported in $\mathrm{Hz}$. Infrared (IR) spectra were recorded as thin films on a Mattson Galaxy Series FTIR 3000 spectrophotometer referenced to a polystyrene standard. Optical rotations were measured on a Jasco DIP 370 digital polarimeter using a quartz cell with $2 \mathrm{~mL}$ capacity and a $10 \mathrm{~cm}$ path length. Mass spectra were recorded at the Harvard University mass spectrometry facility.

Analytical thin layer chromatography (TLC) was performed on Kieselgel $60 \mathrm{~F}_{254}$ glass plates precoated with a $0.25 \mathrm{~mm}$ thickness of silica gel (EM Science). The TLC plates were visualized with UV light and/or by staining with Hanessian solution (a mixture of ceric sulfate and ammonium molybdate in aqueous sulfuric acid). Column chromatography was generally performed according to the method of Still ${ }^{1}$ using Kieselgel 60 (230-400 mesh) silica gel. Unless noted otherwise, all compounds isolated by chromatography were sufficiently pure by ${ }^{1} \mathrm{H}$ NMR analysis for use in

(1) Still, W. C.; Kahn, M.; Mitra, A. J. Org. Chem. 1978, 43, 2923. 
subsequent reactions. The amount of silica gel used for purification was 50-100:1 weight ratio of silica : crude product.

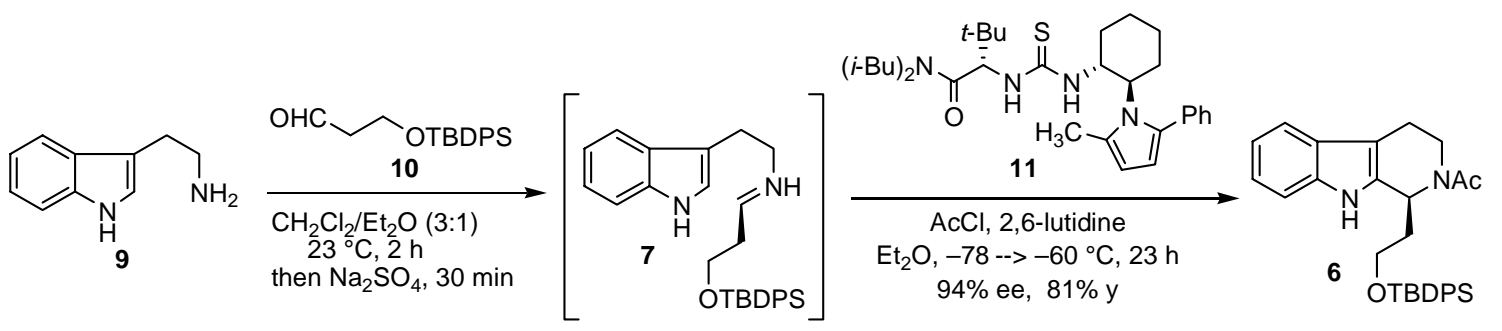

\section{1-((S)-3,4-dihydro-1-(2-(tert-butyl-diphenyl-silanyloxy)ethyl)-1H-pyrido[3,4-b]indol-2(9H)-}

yl)ethanone (6). ${ }^{2}$ Tryptamine (9) (400 mg, $\left.2.5 \mathrm{mmol}\right)$ was dissolved in $\mathrm{CH}_{2} \mathrm{Cl}_{2}(62 \mathrm{~mL})$ and $\mathrm{Et}_{2} \mathrm{O}(31$ $\mathrm{mL}$ ) was added. Aldehyde $\mathbf{1 0}^{3}$ was then added dropwise in $\mathrm{CH}_{2} \mathrm{Cl}_{2}(25 \mathrm{~mL}$ ) over 10 min (Note: best results were achieved when aldehyde $\mathbf{1 0}$ was purified immediately prior to use). The flask containing 10 was rinsed three times with $\mathrm{CH}_{2} \mathrm{Cl}_{2}(3,3,1 \mathrm{~mL})$. These rinses were added and the resulting reaction mixture was stirred for $1.5 \mathrm{~h} . \mathrm{Na}_{2} \mathrm{SO}_{4}(5 \mathrm{~g})$ was then added, and the heterogeneous mixture was stirred vigorously for $30 \mathrm{~min}$. The stirring was then stopped, and the reaction mixture was filtered via cannula to a $200-\mathrm{mL}$ round-bottom flask. The flask originally containing imine 7 was rinsed four times with $\mathrm{CH}_{2} \mathrm{Cl}_{2}(25 \mathrm{~mL}$, then $3 \times 6 \mathrm{~mL})$. This mixture was concentrated yielding 7 as a dark yellow foam, which was used immediately in the acyl-Pictet-Spengler reaction.

Imine 7 was dissolved in $\mathrm{Et}_{2} \mathrm{O}(50 \mathrm{~mL})$. Thiourea catalyst $\mathbf{1 1}^{2}(136 \mathrm{mg}, 0.25 \mathrm{mmol}, 10 \mathrm{~mol} \%)$ was added, and the reaction mixture was cooled to $-78^{\circ} \mathrm{C}$. 2,6-Lutidine $(290 \mu \mathrm{L}, 2.5 \mathrm{mmol})$ was added dropwise, followed by dropwise addition of acetyl chloride $(179 \mu \mathrm{L}, 2.5 \mathrm{mmol})$. The resulting mixture was stirred for $10 \mathrm{~min}$ at $-78{ }^{\circ} \mathrm{C}$ and then transferred to a cryocool bath held at $-60{ }^{\circ} \mathrm{C}$. The mixture was then stirred under positive $\mathrm{N}_{2}$ pressure (balloon) for $22 \mathrm{~h}$ (the reaction mixture became increasingly heterogeneous during this time) at which point the cryocool bath was allowed to warm slowy, over $4.5 \mathrm{~h}$, to $5^{\circ} \mathrm{C}$. The resulting light yellow slurry was concentrated yielding a brownish yellow solid. Purification of this solid by column chromatography (gradient solvent system of $1 \rightarrow 5$ $\rightarrow 10 \%$ ethyl aceate in dichloromethane) provided $6(1.0 \mathrm{~g}, 81 \%)$ as a white foam. This compound exists as a $1.6: 1$ mixture of amide rotamers. The enantiomeric excess was determined to be $94 \%$ by

(2) Taylor, M. S.; Jacobsen, E. N. J. Am. Chem. Soc. 2004, 126, 10558.

(3) Smith, A. B., III.; Safonov, I. G.; Corbett, R. M. J. Am. Chem. Soc. 2002, 124, 11102. 
chiral HPLC according to the previous published method. ${ }^{2}$ The spectroscopic properties of $\mathbf{6}$ were in agreement with the previously published data. ${ }^{2}$
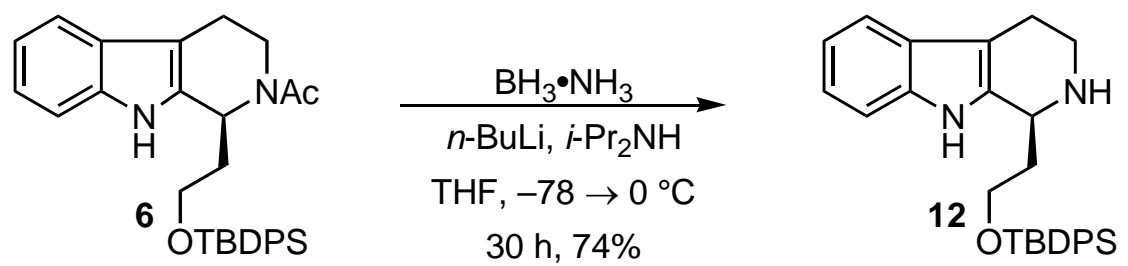

(S)-2,3,4,9-tetrahydro-1-(2-(tert-butyl-diphenyl-silanyloxy)ethyl)-1H-pyrido[3,4-b]indole (12). i- $\operatorname{Pr}_{2} \mathrm{NH}(1.64 \mathrm{~mL}, 11.7 \mathrm{mmol})$ was dissolved in THF $(3.9 \mathrm{~mL})$ and cooled to $-78{ }^{\circ} \mathrm{C} . n$-BuLi $(1.98 \mathrm{M}$ solution in hexanes, $5.4 \mathrm{~mL}, 10.7 \mathrm{mmol}$ ) was added slowly. The resulting white slurry was stirred at $78{ }^{\circ} \mathrm{C}$ for $15 \mathrm{~min}$ and then warmed to $0{ }^{\circ} \mathrm{C}$. After stirring at $0{ }^{\circ} \mathrm{C}$ for $25 \mathrm{~min}, \mathrm{BH}_{3} \cdot \mathrm{NH}_{3}(90 \%, 400 \mathrm{mg}$, $11.6 \mathrm{mmol}$ ) was added in one portion. The frothy white mixture was stirred for $20 \mathrm{~min}$ at $0{ }^{\circ} \mathrm{C}$ and then warmed to $23{ }^{\circ} \mathrm{C}$. The resulting biphasic mixture was stirred vigorously until the viscous lower layer precluded further stirring, providing a solution of lithium amidotrihydroborate. In a separate flask, tetrahydro- $\beta$-carboline 6 was dried azeotropically with benzene. The dry residue was dissolved in THF $(1.05 \mathrm{~mL})$ and cooled to $-78{ }^{\circ} \mathrm{C}$. The top, homogeneous layer of the lithium amidotrihydroborate solution was then added slowly. The resulting light yellow, opaque reaction mixture was stirred at $-78^{\circ} \mathrm{C}$ for $10 \mathrm{~min}$ and warmed to $0^{\circ} \mathrm{C}$. The reaction mixture was transferred to a cold room and stirred in an ice bath at $0{ }^{\circ} \mathrm{C}$ under positive $\mathrm{N}_{2}$ pressure (balloon) for $24 \mathrm{~h}$ at which point only a trace of 6 remained as judged by TLC analysis. Trifluoroethanol $(1.2 \mathrm{~mL})$ was added and the reaction was then diluted with $\mathrm{CH}_{2} \mathrm{Cl}_{2}$. $\mathrm{NaHSO}_{4}(2 \mathrm{M}, 10 \mathrm{~mL})$ was added slowly (CAUTION! Gas evolution) and the biphasic mixture was stirred vigorously for $1 \mathrm{~h}$. The layers were separated and the aqueous layer was extracted with $\mathrm{CH}_{2} \mathrm{Cl}_{2}$. The combined organic phases were washed with saturated $\mathrm{NaHCO}_{3}$ (agitate at least $30 \mathrm{sec}$ in separatory funnel). This aqueous layer was also extracted with $\mathrm{CH}_{2} \mathrm{Cl}_{2}$. The combined organic phases were dried over $\mathrm{Na}_{2} \mathrm{SO}_{4}$, filtered, and concentrated yielding a yellow oil. Purification of this oil by column chromatography $(25 \times 155 \mathrm{~mm}$ silica, gradient solvent mixture of $1 \rightarrow 3 \rightarrow 4 \rightarrow 5 \%$ methanol in dichloromethane) afforded amine 12 as a white foam (100 mg, 74\%): ${ }^{1} \mathrm{H}$ NMR (500 MHz, $\left.\mathrm{CDCl}_{3}\right) \delta 8.76$ (br s, $\left.1 \mathrm{H}\right), 7.72-7.68(\mathrm{~m}, 4 \mathrm{H}), 7.50-7.44$ (m, 3 H), 7.42-7.37 (m, 4 H), 7.15-7.07 (m, 3 H), 4.32-4.28 (m, $1 \mathrm{H}), 4.00-3.92(\mathrm{~m}, 2 \mathrm{H}), 3.33$ (dt, $J=13.2$, 
4.4, 4.4 Hz, $1 \mathrm{H}$ ), 3.06 (ddd, $J=13.2,8.3,5.4 \mathrm{~Hz}, 1 \mathrm{H}), 2.82-2.71$ (m, $2 \mathrm{H}), 2.47-2.25$ (br s, $1 \mathrm{H}$ ), 2.172.09 (m, $1 \mathrm{H}), 1.97-1.91(\mathrm{~m}, 1 \mathrm{H}), 1.15$ (s, $9 \mathrm{H}) ;{ }^{13} \mathrm{C}$ NMR $\left(100 \mathrm{MHz}, \mathrm{CDCl}_{3}\right) \delta 135.8,135.5,135.5$, 135.4, 133.0, 132.9, 130.0, 129.9, 127.9, 127.9, 127.4, 121.3, 119.1, 118.0, 110.8, 108.3, 62.7, 52.1, 42.7, 37.6, 27.0, 22.5, 19.2; $[\alpha]_{\mathrm{D}}{ }^{28}-18.7^{\circ}\left(c^{2} 1.4, \mathrm{CHCl}_{3}\right)$; IR (thin film) 3070, 3051, 2931, 2894, 2858, 1471, 1453, 1428, 1111, 1086, 823, 739, 703, $613 \mathrm{~cm}^{-1}$; HRMS (ES), calcd for $\mathrm{C}_{29} \mathrm{H}_{35} \mathrm{~N}_{2} \mathrm{OSi}[\mathrm{M}+\mathrm{H}]^{+}$ 455.2519 , found $455.2515 \mathrm{~m} / \mathrm{z}$.

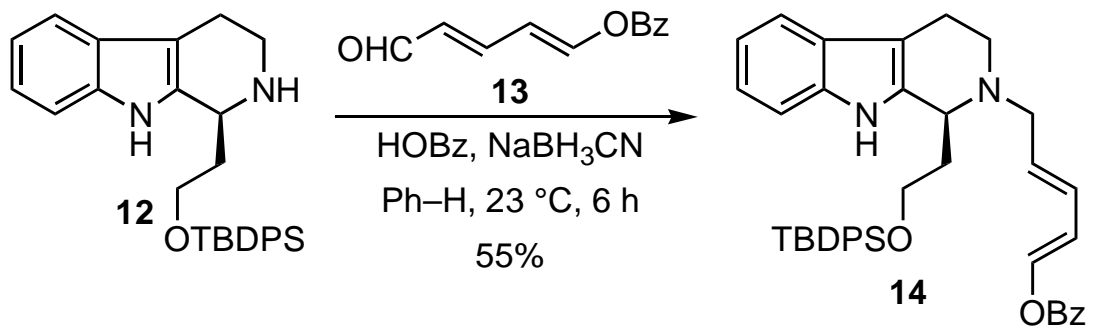

(1E,3E)-5-((S)-3,4-dihydro-1-(2-(tert-butyl-diphenyl-silanyloxy)ethyl)-1H-pyrido[3,4-b]indol2(9H)-yl)penta-1,3-dienyl benzoate (14). Amine 12 (110 mg, $0.24 \mathrm{mmol})$ was dissolved in $\mathrm{Ph}-\mathrm{H}$ $(5.4 \mathrm{~mL})$ and $\mathrm{HOBz}(191 \mathrm{mg}, 1.6 \mathrm{mmol})$ was added followed by $\mathrm{NaBH}_{3} \mathrm{CN}$ (44 mg, $\left.0.7 \mathrm{mmol}\right)$. Aldehyde $13^{4}$ (72 $\mathrm{mg}, 0.36 \mathrm{mmol}$ ) was then added and the clear, heterogeneous reaction mixture turned bright yellow. This mixture was stirred for $6 \mathrm{~h}$ at which point TLC analysis indicated the consumption of aldehyde (the aldehyde co-spots with the product thus the reaction was allowed to proceed until all excess aldehyde had been reduced). Saturated $\mathrm{NaHCO}_{3}$ (ca. $5 \mathrm{~mL}$ ) was added and the biphasic mixture was stirred for $10 \mathrm{~min}$. The biphasic mixture was further diluted with $\mathrm{CH}_{2} \mathrm{Cl}_{2}$ and saturated $\mathrm{NaHCO}_{3}$. The layers were separated and the aqueous layer was extracted twice with $\mathrm{CH}_{2} \mathrm{Cl}_{2}$. The combined organic phases were dried over $\mathrm{Na}_{2} \mathrm{SO}_{4}$, filterd, and concentrated giving a yellow oil. Purification of this material by column chromatography $(25 \times 155 \mathrm{~mm}$ silica, gradient solvent system of $3 \rightarrow 5 \rightarrow 10 \rightarrow 15 \rightarrow 20 \%$ ethyl acetate in hexanes) afforded 14 (85 mg, 55\%) as a clear oil: ${ }^{1} \mathrm{H}$ NMR $\left(500 \mathrm{MHz}, \mathrm{CDCl}_{3}\right) \delta 8.26$ (br s, $\left.1 \mathrm{H}\right), 8.12-8.09$ (m, $\left.2 \mathrm{H}\right)$, 7.74-7.69 (m, $\left.4 \mathrm{H}\right)$, 7.63-7.59 (m, $\left.1 \mathrm{H}\right), 7.58$ $(\mathrm{d}, J=11.7 \mathrm{~Hz}, 1 \mathrm{H}), 7.51-7.39(\mathrm{~m}, 9 \mathrm{H}), 7.17-7.06(\mathrm{~m}, 3 \mathrm{H}), 6.22(\mathrm{q}, J=11.2 \mathrm{~Hz}, 1 \mathrm{H}), 6.18-6.13(\mathrm{~m}$, $1 \mathrm{H}), 5.83$ (ddd, 14.2, 6.8, $6.8 \mathrm{~Hz}, 1 \mathrm{H}), 4.08-4.04$ (m, $1 \mathrm{H}), 3.99-3.94$ (m, $1 \mathrm{H}), 3.86$ (ddd, 11.7, 7.8, $4.4 \mathrm{~Hz}, 1 \mathrm{H}), 3.34-3.24$ (m, 2 H), 3.17 (ddd, 13.2, 8.3, 4.9 Hz, 1 H), 3.00-2.94 (m, 1 H), 2.85 (ddd, 14.7, 9.3, 4.9 Hz, 1 H), 2.59 (dt, 15.1, 3.9, 3.9 Hz, 1 H), 2.11-2.04 (m, 1 H), 1.94-1.87 (m, 1 H), 1.14,

(4) Becher, J. Org. Synth. 1980, 59, 79 
s, $9 \mathrm{H}) ;{ }^{13} \mathrm{C} \mathrm{NMR}\left(100 \mathrm{MHz}, \mathrm{CDCl}_{3}\right) \delta 163.4,138.1,135.6,135.6,135.5,134.8,133.6,133.5,133.2$, $132.0,129.9,128.9,128.5,127.9,127.9,127.3,127.2,121.2,119.0,118.0,115.7,110.7,107.3,62.1$, 55.4, 54.0, 45.2, 36.8, 27.0, 19.3, 18.1; $[\alpha]_{\mathrm{D}}^{28}-38.9^{\circ}$ (c 1.5, $\mathrm{CHCl}_{3}$ ); IR (thin film) 3423, 2932, 2890, 2858, 1733, 1465, 1452, 1428, 1315, 1298, 1264, 1157, 1114, 1091, 979, 740, 706, $506 \mathrm{~cm}^{-1}$; HRMS (ES), calcd for $\mathrm{C}_{41} \mathrm{H}_{45} \mathrm{~N}_{2} \mathrm{O}_{3} \mathrm{Si}[\mathrm{M}+\mathrm{H}]^{+} \quad 641.3199$, found $641.3201 \mathrm{~m} / \mathrm{z}$.
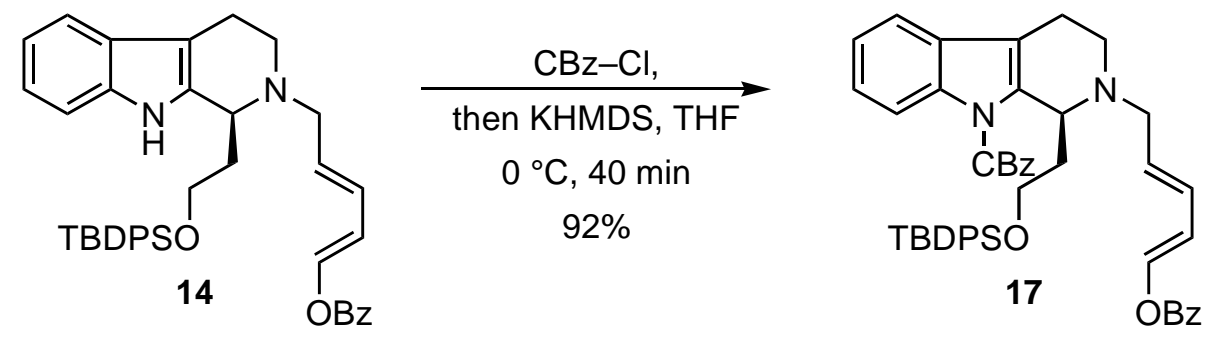

\section{(1E,3E)-5-((S)-3,4-dihydro-1-(2-(tert-butyl-diphenyl-silanyloxy)ethyl)-1H-pyrido[3,4-b]indol-} 2(9-carbobenzyloxy)-yl)penta-1,3-dienyl benzoate (17). Amine 14 (115 mg, $0.18 \mathrm{mmol}$ ) was dried azeotropically by evaporation of a benzene solution. This dry material was dissolved in THF (3.6 mL) and cooled to $0{ }^{\circ} \mathrm{C}$. $\mathrm{CBz}-\mathrm{Cl}(36 \mu \mathrm{L}, 0.26 \mathrm{mmol})$ was added, followed by KHMDS (1 M solution prepared by dissolving $1 \mathrm{mmol}$ solid KHMDS in $1 \mathrm{~mL}$ THF, $210 \mu \mathrm{L}, 0.21 \mathrm{mmol}$ ). The resulting light yellow reaction mixture was stirred for $15 \mathrm{~min}$, at which point additional $\mathrm{CBz}-\mathrm{Cl}$ ( $36 \mathrm{uL}, 0.26 \mathrm{mmol}$ ) was added followed by KHMDS ( $1 \mathrm{M}$ in THF, $210 \mu \mathrm{L}, 0.21 \mathrm{mmol}$ ). The reaction mixture was stirred for an additional $25 \mathrm{~min}$ at which point only a trace of $\mathbf{1 3}$ remained as judged by TLC analysis. Saturated $\mathrm{NH}_{4} \mathrm{Cl}$ was added, the ice bath was removed, and the biphasic mixture was stirred vigorously for $5 \mathrm{~min}$. The reaction mixture was then diluted further with ethyl acetate and saturated $\mathrm{NH}_{4} \mathrm{Cl}$. The layers were separated, and the aqueous layer was extracted with ethyl acetate. The combined organic phases were washed with saturated $\mathrm{NaHCO}_{3}$, dried over $\mathrm{Na}_{2} \mathrm{SO}_{4}$, and concentrated yielding a yellow oil. Purification of this oil by column chromatography $(25 \times 150 \mathrm{~mm}$ silica, gradient solvent system of $1 \rightarrow 7 \rightarrow 15 \%$ ethyl acetate in hexanes) afforded $N$-CBz indole $\mathbf{1 7}$ as a clear oil. This material was contaminated with an inseparable impurity derived from addition of KHMDS to CBz-Cl $\left(\mathrm{BnO}_{2} \mathrm{CNH}(\mathrm{TMS})\right)$. The corrected yield of 15 was $129 \mathrm{mg}(92 \%)$. Data for 15: ${ }^{1} \mathrm{H}$ NMR (500 MHz, $\left.\mathrm{CDCl}_{3}\right) \delta 8.16-8.10(\mathrm{~m}, 2 \mathrm{H})$, 7.75-7.68 (m, $\left.3 \mathrm{H}\right)$, 7.66-7.60 (m, 1 H), 7.54-7.48 (m, $\left.2 \mathrm{H}\right)$, 7.46-7.30 (m, $13 \mathrm{H}), 7.29-7.21(\mathrm{~m}, 3 \mathrm{H}), 6.17$ (t, $J=11.2 \mathrm{~Hz}, 1 \mathrm{H}), 6.05$ (dd, $J=15.1,11.2 \mathrm{~Hz}, 1 \mathrm{H}), 5.68$ (ddd, $J$ $=13.7,6.4,6.4 \mathrm{~Hz}, 1 \mathrm{H}$ ), 4.27 (br d, $J=8.8 \mathrm{~Hz}, 1 \mathrm{H}$ ), 3.90-3.80 (m, $2 \mathrm{H}$ ), 3.21 (dddd, $J=14.2,14.2$, 14.2, $6.4 \mathrm{~Hz}, 2 \mathrm{H}), 3.10-3.02$ (m $1 \mathrm{H}), 2.91$ (dd, $J=14.2,6.4 \mathrm{~Hz}, 1 \mathrm{H}), 2.76$ (ddd, $J=17.1,11.7,5.9$ 
$\mathrm{Hz}, 1 \mathrm{H}), 2.41(\mathrm{dd}, J=16.6,4.9 \mathrm{~Hz}, 1 \mathrm{H}), 2.18-2.11(\mathrm{~m}, 1 \mathrm{H}), 1.87-1.81(\mathrm{~m}, 1 \mathrm{H}), 1.07(\mathrm{~s}, 9 \mathrm{H}) ;{ }^{13} \mathrm{C}$ NMR (100 MHz, $\left.\mathrm{CDCl}_{3}\right) \delta 163.5,151.3,137.9,136.7,136.5,135.8,135.6,135.0,134.4,134.3,133.6$, 132.6, 130.0, 129.6, 129.4, 129.4, 128.9, 128.7, 128.7, 128.6, 128.6, 128.5, 128.1, 128.0, 127.6, 127.5, $126.5,124.1,122.9,117.8,115.9,115.8,114.8,77.2,68.5,66.9,62.1,55.3,54.8,41.0,37.1,26.9$, 19.3, 16.6; $\left.[\alpha]_{\mathrm{D}}{ }^{24}-17.4^{\circ}(c) .7, \mathrm{CHCl}_{3}\right)$; IR (thin film) 3070, 3034, 2957, 2933, 2898, 2858, 1729, 1455, 1429, 1392, 1361, 1321, 1297, 1254, 1211, 1179, 1142, 1114, 1088, 1069, 1021, 978, 865, 849, 744, $703 \mathrm{~cm}^{-1}$; HRMS (ES) calcd for $\mathrm{C}_{49} \mathrm{H}_{51} \mathrm{~N}_{2} \mathrm{O}_{5} \mathrm{Si}[\mathrm{M}+\mathrm{H}]^{+} 775.3567$, found $775.3553 \mathrm{~m} / \mathrm{z}$.
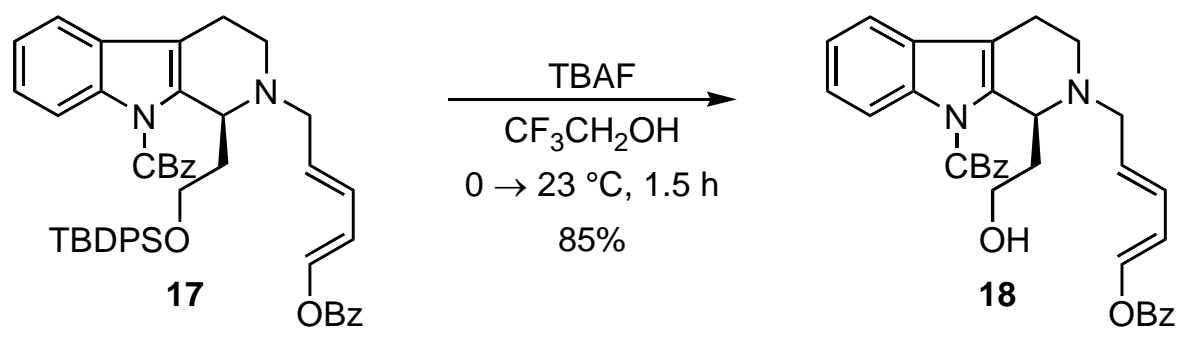

\section{(1E,3E)-5-((S)-3,4-dihydro-1-(2-hydroxyethyl)-1H-pyrido[3,4-b]indol-2(9-carbobenzyloxy)-}

yl)penta-1,3-dienyl benzoate (18). Trifluoroethanol $(4.7 \mathrm{~mL})$ was added to a flask containing silyl ether $17(105 \mathrm{mg}, 0.14 \mathrm{mmol})$ and the mixture was stirred vigorously for ca. $15 \mathrm{~min}$ (17 is only partially soluble in trifluoroethanol). The resulting suspension was cooled to $0{ }^{\circ} \mathrm{C}$ and TBAF $(1 \mathrm{M}$ solution in THF, $840 \mu \mathrm{L}, 0.84 \mathrm{mmol}$ ) was added. The resulting mixture was alloed to warm to $23{ }^{\circ} \mathrm{C}$ and then stirred for $1.25 \mathrm{~h}$ at which point 17 was consumed as judged by TLC analysis. The reaction mixture was poured into saturated $\mathrm{NH}_{4} \mathrm{Cl}$ and quickly diluted with ethyl acetate (Note: It was necessary to use saturated $\mathrm{NH}_{4} \mathrm{Cl}$ for the aqueous workup. Complete decomposition was observed when saturated $\mathrm{NaHCO}_{3}$ was used). This biphasic mixture was agitated in the separatory funnel, the layers were separated, and the aqueous layer was extracted with ethyl acetate. The combined organic phases were washed with saturated sodium chloride, dried over $\mathrm{Na}_{2} \mathrm{SO}_{4}$, filtered, and concentrated yielding a yellow oil contaminated with trifluoroethanol. Excess trifluoroethanol was removed via high vacuum. The resulting crude oil was purified by column chromatography $(25 \times 160 \mathrm{~mm}$ silica, gradient solvent system of $1 \rightarrow 7 \rightarrow 15 \rightarrow 20 \%$ acetone in hexanes) and alcohol 16 was isolated as a clear oil. This material was contaminated with $\mathrm{BnOC}(\mathrm{O}) \mathrm{NH}_{2}$. The corrected yield of $\mathbf{1 8}$ was $64 \mathrm{mg}$ (85\%). Data for 17: ${ }^{1} \mathrm{H}$ NMR (500 MHz, $\left.\mathrm{CDCl}_{3}\right) \delta 8.16(\mathrm{~d}, J=7.8 \mathrm{~Hz}, 1 \mathrm{H}), 8.14-8.10(\mathrm{~m}, 2 \mathrm{H}), 7.64-$ 7.59 (m, 2 H), 7.52-7.36 (m, 7 H), 7.32-7.25 (m, 3 H), 6.27 (t, $J=11.2 \mathrm{~Hz}, 1 \mathrm{H}), 6.15$ (dd, $J=15.1$, 
$10.7 \mathrm{~Hz}, 1 \mathrm{H}), 5.81$ (ddd, $J=14.7,6.8,6.8 \mathrm{~Hz}, 1 \mathrm{H}), 5.43(\mathrm{~d}, J=11.7 \mathrm{~Hz}, 1 \mathrm{H}$, A of AB system), 5.38 (d, $J=11.7 \mathrm{~Hz}, 1 \mathrm{H}$, B of AB system), 4.45 (br d, $J=9.78 \mathrm{~Hz}, 1 \mathrm{H}), 3.64-3.54$ (m, $2 \mathrm{H}), 3.42-3.28$ (m, $3 \mathrm{H}$ ), 3.07 (dd, $J=14.2,6.4 \mathrm{~Hz}, 1 \mathrm{H}$ ), 2.82 (ddd, $J=16.6,11.7,6.4 \mathrm{~Hz}, 1 \mathrm{H}$ ), 2.57 (dd, $J=16.6,4.9$ $\mathrm{Hz}, 1 \mathrm{H}), 2.02-1.91(\mathrm{~m}, 1 \mathrm{H}), 1.89-1.83(\mathrm{~m}, 1 \mathrm{H}) ;{ }^{13} \mathrm{C} \mathrm{NMR}\left(100 \mathrm{MHz}, \mathrm{CDCl}_{3}\right) \delta 163.4,151.2,138.6$, $135.8,134.7,134.5,133.7,130.6,130.0,129.3,129.0,129.0,128.8,128.7,128.6,128.5,128.2$, 128.1, 124.6, 123.2, 118.0, 115.9, 115,4, 114.9, 69.0, 63.5, 59.2, 55.1, 41.3, 34.3, 16.2; $[\alpha]_{\mathrm{D}}\left(c, \mathrm{CHCl}_{3}\right) ; \mathrm{IR}$ (thin film) 3285, 3071, 3035, 2949, 2848, 1730, 1456, 1395, 1350, 1323, 1263, 1208, 1143, 1128, 1066, 1040, 1021, 979, 751, $709 \mathrm{~cm}^{-1}$; HRMS (ES) calcd for $\mathrm{C}_{33} \mathrm{H}_{33} \mathrm{~N}_{2} \mathrm{O}_{5}[\mathrm{M}+\mathrm{H}]^{+} 537.2389$, found $537.2386 \mathrm{~m} / \mathrm{z}$.
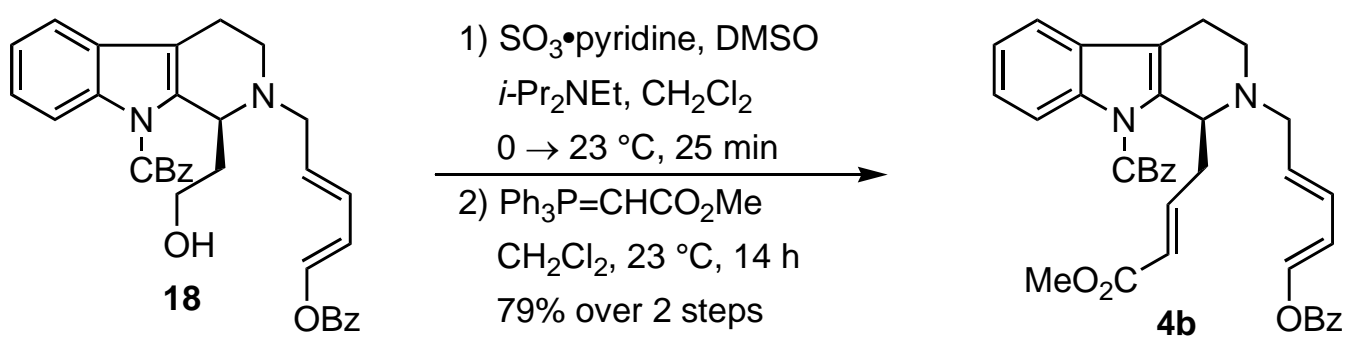

\section{(1E,3E)-5-((S)-1-((E)-3-(methoxycarbonyl)allyl)-3,4-dihydro-1H-pyrido[3,4-b]indol-2(9-}

carbobenzyloxy)-yl)penta-1,3-dienyl benzoate (4b). Alcohol 18 (57 $\mathrm{mg}, 0.11 \mathrm{mmol})$ was dried azeotropically by evaporation of a benzene solution. The solid residue was dissolved in $\mathrm{CH}_{2} \mathrm{Cl}_{2}(3.7$ $\mathrm{mL})$ and $i$ - $\operatorname{Pr}_{2} \mathrm{NEt}(160 \mu \mathrm{L}, 0.92 \mathrm{mmol})$ was added followed by DMSO $(130 \mu \mathrm{L}, 1.83 \mathrm{mmol})$. The mixture was cooled to $0{ }^{\circ} \mathrm{C}$ and $\mathrm{SO}_{3} \cdot$ pyridine $(78 \mathrm{mg}, 0.49 \mathrm{mmol}$ ) was added in one portion. The resulting mixture was stirred for $25 \mathrm{~min}$ at which point TLC analysis indicated the complete consumption of 18. The reaction mixture was diluted with saturated $\mathrm{NaHCO}_{3}$, the ice bath was removed, and the biphasic mixture was stirred vigorously for $10 \mathrm{~min}$. The mixture was further diluted with saturated $\mathrm{NaHCO}_{3}$ and ethyl acetate. The layers were separated and the aqueous layer was extracted with ethyl acetate. The combined organic phases were washed with saturated sodium chloride, dried over $\mathrm{Na}_{2} \mathrm{SO}_{4}$, filtered, and concentrated yielding a yellow, odorous oil. Excess pyridine was removed from this crude oil by coevaporation with benzene. The resulting pyridine-free residue was used immediately in the following olefination reaction.

The crude aldehyde (prepared above, theoretically $0.11 \mathrm{mmol}$ ) was dissolved in $\mathrm{CH}_{2} \mathrm{Cl}_{2}$ and methyl(triphenylphosphoranylidene)acetate $(78 \mathrm{mg}, 0.23 \mathrm{mmol})$ was added. The resulting orange 
reaction mixture was stirred for $1 \mathrm{~h}$ at which point additional methyl(triphenylphosphoranylidene)acetate $(37 \mathrm{mg}, 0.11 \mathrm{mmol})$ was added. The reaction mixture was stirred for $11.5 \mathrm{~h}$, and the recharging process was repeated as above. The reaction mixture was stirred for an additional $2.5 \mathrm{~h}$ at which point all of the aldehyde had been consumed as judged by TLC analysis. This mixture was partially concentrated and the resulting maroon oil was purified by column chromatography (25x120mm silica, gradient solvent system of $5101520 \%$ acetone in hexanes), and triene $4 \mathbf{b}$ (51 mg, 79\% over 2 steps) was isolated as a white solid. An analytical sample of $\mathbf{4 b}$ was prepared by preparative TLC $\left(250 \mu \mathrm{M}\right.$ silica plate, $40 \%$ ethyl acetate in hexanes): ${ }^{1} \mathrm{H}$ NMR (500 $\left.\mathrm{MHz} \mathrm{CDCl}_{3}\right) \delta 8.15(\mathrm{br} \mathrm{d}, J=7.8 \mathrm{~Hz}, 1 \mathrm{H}), 8.13-8.10(\mathrm{~m}, 2 \mathrm{H}), 7.64-7.59(\mathrm{~m}, 1 \mathrm{H}) 7.58(\mathrm{~d}, J=12.2$ $\mathrm{Hz}, 1 \mathrm{H}), 7.51-7.36(\mathrm{~m}, 7 \mathrm{H}), 7.31-7.24(\mathrm{~m}, 3 \mathrm{H}), 6.94(\mathrm{ddd}, J=15.6,7.3,7.3 \mathrm{~Hz}, 1 \mathrm{H}), 6.26(\mathrm{t}, J=$ $11.2 \mathrm{~Hz}, 1 \mathrm{H}), 6.15(\mathrm{dd}, J=14.6,10.7 \mathrm{~Hz}, 1 \mathrm{H}), 5.79-5.72(\mathrm{~m}, 1 \mathrm{H}), 5.77$ (t, $J=15.6 \mathrm{~Hz}, 1 \mathrm{H}), 5.44(\mathrm{~d}$, $J=12.2 \mathrm{~Hz}, 1 \mathrm{H}$, A of AB system), 5.40 (d, $J=12.2 \mathrm{H}, 1 \mathrm{H}, \mathrm{B}$ of AB system), 4.38 (br d, $J=7.8 \mathrm{~Hz}, 1$ H), $3.77(\mathrm{~s}, 3 \mathrm{H}), 3.32(\mathrm{dd}, J=14.2,5.9 \mathrm{~Hz}, 1 \mathrm{H}), 3.26-3.16(\mathrm{~m}, 2 \mathrm{H}), 3.04(\mathrm{dd}, J=14.2,5.9 \mathrm{~Hz}, 1 \mathrm{H})$, 2.86-2.76 (m, $1 \mathrm{H}), 2.69-2.62(\mathrm{~m}, 1 \mathrm{H}), 2.53-2.45(\mathrm{~m}, 2 \mathrm{H}) ;{ }^{13} \mathrm{C}$ NMR $\left(100 \mathrm{MHz}, \mathrm{CDCl}_{3}\right) \delta 167.0$, $163.4,151.3,147.4,138.1,135.8,135.5,134.6,133.6,131.9,130.0,129.4,128.9,128.8,128.6,127.0$, $124.5,123.1,121.7,118.1,115.9,115.8,115.5,69.0,56.6,55.3,51.4,41.5,36.9,16.8 ;[\alpha]_{\mathrm{D}}{ }^{27}-2.2^{\circ}(c$ $2.4, \mathrm{CHCl}_{3}$ ); IR (thin film) 3072, 3034, 2996, 2948, 2846, 1728, 1658, 1456, 1436, 1394, 1351, 1323, 1264, 1208, 1158, 1143, 1120, 1021, 980, 751, $709 \mathrm{~cm}^{-1}$; HRMS (ES) calcd for $\mathrm{C}_{36} \mathrm{H}_{35} \mathrm{~N}_{2} \mathrm{O}_{6}[\mathrm{M}+\mathrm{H}]^{+}$ 591.2495 , found $591.2486 \mathrm{~m} / \mathrm{z}$.

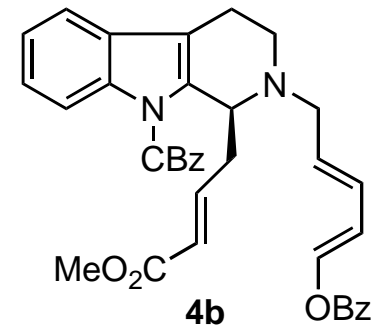

$$
\begin{gathered}
\underset{\mathrm{Sc}(\mathrm{OTf})_{3}(2 \rightarrow 4 \text { equiv })}{\stackrel{\mathrm{CH}_{3} \mathrm{CN}, 23{ }^{\circ} \mathrm{C}, 67 \mathrm{~h}}{\longrightarrow}} \\
87 \%, \mathrm{dr}>20: 1
\end{gathered}
$$

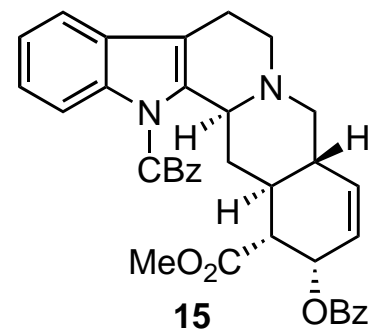

(3E,5E,8S,9R,10Z,12E)-11-bromo-9-(tert-butyl-dimethyl-silanyloxy)-14-hydroxy-8-[(6-Deoxy2,3,4-trimethyl-O-methyl- $\alpha$-L-mannopyranosyl)oxy]-tetradeca-3,5,10,12-tetraen-2-one (15). Triene 4b (47 mg, $0.08 \mathrm{mmol})$ was dried azeotropically by evaporation of a benzene solution. The resulting solid residue was dissolved in $\mathrm{CH}_{3} \mathrm{CN}(2.6 \mathrm{~mL})$ and $\mathrm{Sc}(\mathrm{OTf})_{3}(79 \mathrm{mg}, 0.16 \mathrm{mmol})$ was added. The reaction mixture was stirred for $15 \mathrm{~h}$ at which point additional $\mathrm{Sc}(\mathrm{OTf})_{3}(39 \mathrm{mg}, 0.08$ 
mmol) was added. The reaction mixture was then stirred an additional $13 \mathrm{~h}$ at which point $\mathrm{Sc}(\mathrm{OTf})_{3}$ (39 $\mathrm{mg}, 0.08 \mathrm{mmol}$ ) was again added. This mixture was stirred for $15 \mathrm{~h}$ at which point $\mathbf{4 b}$ had been consumed as judged by TLC analysis. The reaction mixture was poured into a biphasic mixture of saturated $\mathrm{NaHCO}_{3}$ and $\mathrm{CH}_{2} \mathrm{Cl}_{2}$. This biphasic mixture was shaken in the separatory funnel, additional saturated $\mathrm{NaHCO}_{3}$ was added, the mixture was further agitated, additional saturated $\mathrm{NaHCO}_{3}$ was added, and the mixture was shaken again. The aqueous layer was then extracted three times with $\mathrm{CH}_{2} \mathrm{Cl}_{2}$. The combined organic phases were dried over $\mathrm{Na}_{2} \mathrm{SO}_{4}$, filtered, and concentrated yielding a yellow oil. Purification of this oil by column chromatography $(25 \times 150 \mathrm{~mm}$ silica, gradient solvent system of $5 \rightarrow 10 \rightarrow 15 \rightarrow 20 \%$ acetone in hexanes) afforded 15 (41 mg, 87\%) as a white solid: ${ }^{1} \mathrm{H}$ NMR (500 MHz, $\left.\mathrm{CDCl}_{3}\right) \delta 8.15$ (br d, $\left.7.8 \mathrm{~Hz}, 1 \mathrm{H}\right), 8.07-8.04$ (m, 2 H), 7.60-7.57 (m, 2 H), 7.56-7.52 (m, $1 \mathrm{H}), 7.42-7.33(\mathrm{~m}, 5 \mathrm{H}), 7.29-7.21(\mathrm{~m}, 3 \mathrm{H}), 6.02(\mathrm{ddd}, J=9.8,4.4,2.9 \mathrm{~Hz}, 1 \mathrm{H}), 5.83(\mathrm{~d}, J=9.8$ $\mathrm{Hz}, 1 \mathrm{H}), 5.75$ (br t, $J=4.4 \mathrm{~Hz}, 1 \mathrm{H}), 5.73$ (d, $J=12.0 \mathrm{~Hz}, 1 \mathrm{H}$, A of AB system), 5.47 (d, $J=12.0 \mathrm{~Hz}$, $1 \mathrm{H}, \mathrm{B}$ of AB system), 4.22 (br d, $J=9.8 \mathrm{~Hz}, 1 \mathrm{H}), 3.58$ (s, $3 \mathrm{H}), 3.20-3.14$ (m, $2 \mathrm{H}), 2.86-2.70$ (m, 5 H), 2.69-2.64 (m, $1 \mathrm{H}), 2.29$ (br t, $J=10.7 \mathrm{~Hz}, 1 \mathrm{H}), 2.16$ (dddd, $J=11.7,11.7,11.7,2.9 \mathrm{~Hz}, 1 \mathrm{H})$, $1.30(\mathrm{ddd}, J=11.7,11.7,11.7 \mathrm{~Hz}, 1 \mathrm{H}) ;{ }^{13} \mathrm{C} \mathrm{NMR}\left(100 \mathrm{MHz}, \mathrm{CDCl}_{3}\right) \delta 170.8,165.8,151.4,136.8$, 136.2, 135.3, 134.4, 133.1, 130.1, 129.7, 129.2, 129.0, 128.7, 128.6, 128.4, 124.7, 124.3, 122.9, 118.0, $116.5,115.4,68.7,67.4,59.6,59.2,51.7,49.1,47.2,37.2,36.4,31.4,22.4 ;[\alpha]_{\mathrm{D}}{ }^{32}+139.0^{\circ}$ (c 2.0 , $\mathrm{CHCl}_{3}$ ); IR (thin film) 3064, 3033, 2995, 2949, 2916, 2848, 2809, 1730, 1457, 1436, 1394, 1350, $1315,1269,1212,1162,1112,1070,1026,742,712 \mathrm{~cm}^{-1}$; HRMS (ES) calcd for $\mathrm{C}_{36} \mathrm{H}_{35} \mathrm{~N}_{2} \mathrm{O}_{6}[\mathrm{M}+$ $\mathrm{H}]^{+}$591.2495, found $591.2480 \mathrm{~m} / \mathrm{z}$.
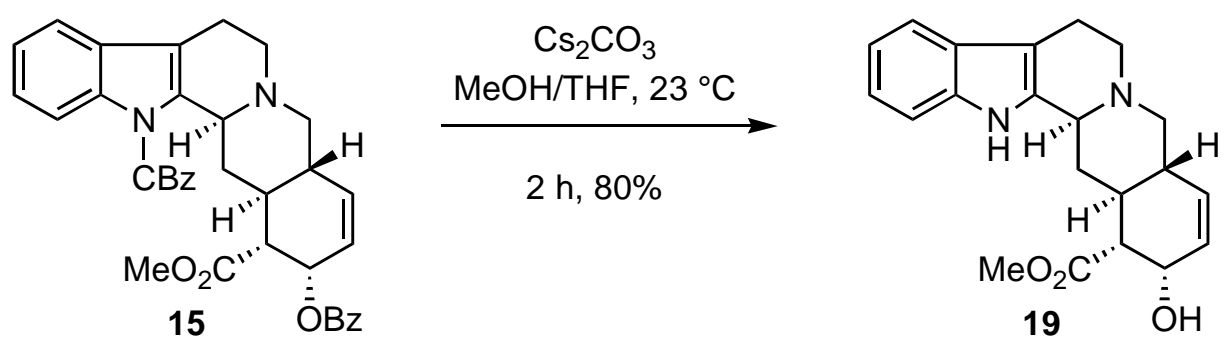

(2Z,4E,6Z,8R,9S,11E,13E)-6-bromo-8-(tert-butyl-dimethyl-silanyloxy)-15-oxo-9-[(6-deoxy-2,3,4trimethyl-O-methyl- $\alpha$-L-mannopyranosyl)oxy]-hexadeca-2,4,6,11,13-pentaenoic acid methyl ester (19). Pentacycle $15(9 \mathrm{mg}, 0.015 \mathrm{mmol})$ was dried azeotropically by evaporation of a benzene solution. The resulting dry residue was dissolved in THF $(220 \mu \mathrm{L})$ and $\mathrm{MeOH}(180 \mu \mathrm{L})$ added. 
$\mathrm{Cs}_{2} \mathrm{CO}_{3}(12 \mathrm{mg}, 0.037 \mathrm{mmol})$ was then added and the resulting heterogeneous mixture was stirred vigorously for $2 \mathrm{~h}$ at which point the mixture was homogeneous. TLC analysis of this mixture indicated the complete consumption of 15. The reaction mixture was diluted with saturated $\mathrm{NH}_{4} \mathrm{Cl}$ and transferred to a separatory funnel. The reaction flask was rinsed with ethyl acetate and these rinses were added to the separatory funnel. The mixture was shaken, the layers were separated, and the aqueous layer was extracted twice with ethyl acetate. The combined organic phases were washed with saturated $\mathrm{NaHCO}_{3}$ (shaken $\geq 1 \mathrm{~min}$ ) then washed with saturated $\mathrm{NaCl}$, dried over $\mathrm{Na}_{2} \mathrm{SO}_{4}$, filtered, and concentrated yielding a yellow oil. Purification of this oil by preparative TLC (250 uM silica plate, 7 \% $\mathrm{MeOH}$ in $\mathrm{CH}_{2} \mathrm{Cl}_{2}$ ) afforded $15(4.3 \mathrm{mg}, 80 \%)$ as a clear oil: ${ }^{1} \mathrm{H} \mathrm{NMR}\left(500 \mathrm{MHz}, \mathrm{CDCl}_{3}\right) \delta 7.83(\mathrm{br}$ s, $1 \mathrm{H}), 7.47(\mathrm{~d}, J=7.3 \mathrm{~Hz}, 1 \mathrm{H}), 7.30$ (d, $J=7.8 \mathrm{~Hz}, 1 \mathrm{H}), 7.16-7.12$ (m, $1 \mathrm{H}), 7.11-7.07$ (m, $1 \mathrm{H})$, $5.91(\mathrm{ddd}, J=9.8,4.9,2.9 \mathrm{~Hz}, 1 \mathrm{H}), 5.74$ (d, $J=9.8 \mathrm{~Hz}, 1 \mathrm{H}), 4.44$ (br t, $J=3.9 \mathrm{~Hz}, 1 \mathrm{H}), 3.81$ (s, 3 H), 3.43, (br d, $J=9.8 \mathrm{~Hz}, 1 \mathrm{H}), 3.15-3.09$ (m, 2 H), 3.00 (dddd, $J=15.1,8.8,5.9,2.4 \mathrm{~Hz}, 1 \mathrm{H}), 2.77-$ $2.65(\mathrm{~m}, 2 \mathrm{H}), 2.62(\mathrm{dd}, J=11.7,3.9, \mathrm{~Hz}, 1 \mathrm{H}), 2.43(\mathrm{dt}, J=12.2,2.9 \mathrm{~Hz}, 1 \mathrm{H}), 2.30(\mathrm{ddd}, J=11.7$, 11.7, 11.7, $1 \mathrm{H}), 2.30-2.23$ (m, $1 \mathrm{H}), 1.93$ (dddd, $J=11.7,11.7,11.7,2.9 \mathrm{~Hz}, 1 \mathrm{H}), 1.39$ (ddd, $J=11.7$, 11.7, $11.7 \mathrm{~Hz}, 1 \mathrm{H})$; ${ }^{13} \mathrm{C} \mathrm{NMR}\left(100 \mathrm{MHz}, \mathrm{CDCl}_{3}\right) \delta 173.3,136.0,134.3,132.5,127.9,127.3,121.4$, $119.4,118.1,110.8,108.1,65.1,60.1,59.8,52.9,51.8,50.5,40.9,34.9,33.0,21.8 ;[\alpha]_{\mathrm{D}}{ }^{28}+44.2^{\circ}(c$ 0.21, $\mathrm{CHCl}_{3}$ ); IR (thin film) 3377, 3360, 3054, 3024, 2948, 2916, 2851, 2811, 2759, 1727, 1452, 1438, $1378,1353,1323,1293,1265,1219,1201,1176,1156,1082,1046,1004,740 \mathrm{~cm}^{-1}$; HRMS (ES) calcd for $\mathrm{C}_{21} \mathrm{H}_{25} \mathrm{~N}_{2} \mathrm{O}_{3}[\mathrm{M}+\mathrm{H}]^{+} 353.1865$ found $353.1852 \mathrm{~m} / \mathrm{z}$.
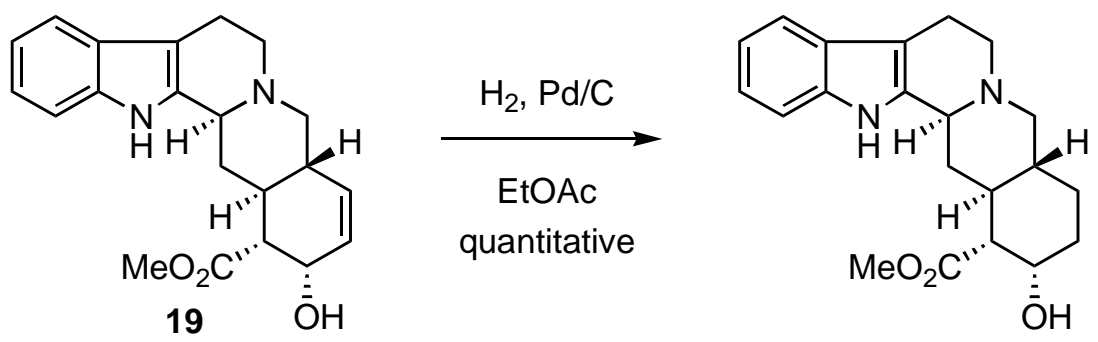

(+)-yohimbine (1)

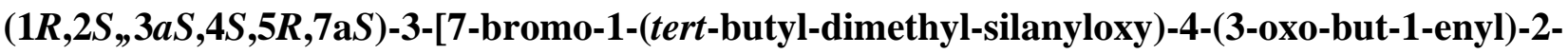
[(6-Deoxy-2,3,4-trimethyl-O-methyl- $\alpha$-L-mannopyranosyl)oxy]--2,3,3a,4,5,7a-hexahydro-1Hinden-5-yl]-acrylic acid methyl ester (1). Olefin 17 was dissolved in ethyl acetate, transferred to a 5 $\mathrm{mL}$ round-bottom flask, and concentrated. The residue was redissolved in ethyl acetate (HPLC/GC grade, $600 \mu \mathrm{L}$ ) and $10 \% \mathrm{Pd} / \mathrm{C}$ was added. The reaction vessel containing 17 in ethyl acetate was then 
evacuated until gentle bumping of the solvent was observed, and then backfilled with $\mathrm{H}_{2}$. This process was repeated eleven times. The reaction was then stirred under $\mathrm{H}_{2}$ (balloon pressure) for $14 \mathrm{~h}$ at which point the reaction was diluted with ethyl acetate (ca. $1.5 \mathrm{~mL}$ ), followed by saturated $\mathrm{NaHCO}_{3}$ (ca. 1.5 $\mathrm{mL}$ ). The biphasic mixture was stirred vigorously for $5 \mathrm{~min}$, transferred to a separatory funnel, further dlited with ethyl acetate and saturated $\mathrm{NaHCO}_{3}$ and shaken for $1 \mathrm{~min}$. The layers were separated and the aqueous layer was extracted with ethyl acetate. The combined organic phases were washed with saturated $\mathrm{NaCl}$, dried over a mixture of $\mathrm{Na}_{2} \mathrm{SO}_{4}$ and $\mathrm{K}_{2} \mathrm{CO}_{3}$, filtered, and concentrated yielding (+)yohimbine (1) (4 mg, quantitative) as white solid. An analytical sample of 1 was prepared by preparative TLC (10\% isopropanol in $\left.\mathrm{CH}_{2} \mathrm{Cl}_{2}\right):{ }^{1} \mathrm{H}$ NMR $\left(500 \mathrm{MHz}, \mathrm{CDCl}_{3}\right) \delta 7.73(\mathrm{br} \mathrm{s}, 1 \mathrm{H}) 7.47$ (d, $J=7.8 \mathrm{~Hz}, 1 \mathrm{H}), 7.31(\mathrm{~d}, J=7.8 \mathrm{~Hz}, 1 \mathrm{H}), 7.16-7.11(\mathrm{~m}, 1 \mathrm{H}), 7.11-7.06(\mathrm{~m}, 1 \mathrm{H}), 4.23$ (br s, $1 \mathrm{H})$, 3.82 (s, $3 \mathrm{H}), 3.35$ (br d, $J=10.7 \mathrm{~Hz}, 1 \mathrm{H}), 3.09$ (dd, $J=10.7,5.4 \mathrm{~Hz}, 1 \mathrm{H}), 3.04-2.95$ (m, $1 \mathrm{H}), 2.96$ (dd, $J=11.2,2.9 \mathrm{~Hz}, 1 \mathrm{H}), 2.91$ (br s, 1 H), 2.75-2.69 (m, $1 \mathrm{H}$ ), 2.63 (ddd, $J=11.2,11.2,4.4 \mathrm{~Hz}, 1 \mathrm{H}$ ), 2.36 (dd, $J=11.7,2.4 \mathrm{~Hz}, 1 \mathrm{H}), 2.29-2.23$ (m, $1 \mathrm{H}), 2.08-1.96$ (m, $3 \mathrm{H}), 1.64-1.52$ (m, $3 \mathrm{H}), 1.47-1.42$ $(\mathrm{m}, 1 \mathrm{H}), 1.38$ (ddd, $J=11.2,11.2,11.2 \mathrm{~Hz}, 1 \mathrm{H}) ;{ }^{13} \mathrm{C} \mathrm{NMR}\left(100 \mathrm{MHz}, \mathrm{CDCl}_{3}\right) \delta 175.6,136.0,134.3$, 127.4, 121.4, 119.4, 118.1, 110.7, 108.3, 66.9, 61.3, 59.9, 52.9, 52.3, 52.0, 40.7, 36.7, 34.3, 31.4, 23.3, 21.7; $[\alpha]_{\mathrm{D}}{ }^{30}+40.0^{\circ}$ (c 0.28, EtOH); IR (thin film) 3529, 2918, 2874, 2851, 1726, 1452, 1436, 1323 , $1295,1270,1207,1149,1112,1096,1006,969,739,720 \mathrm{~cm}^{-1}$; HRMS (ES) calcd for $\mathrm{C}_{21} \mathrm{H}_{27} \mathrm{~N}_{2} \mathrm{O}_{3}$ [M $+\mathrm{H}]^{+} 355.2022$ found $355.2014 \mathrm{~m} / \mathrm{z}$. 
Comparison of ${ }^{1} \mathrm{H}$ NMR Spectral Data for Yohimbine (1)

\begin{tabular}{|c|c|c|}
\hline Aube & This Work & Natural" \\
\hline $7.77(5,1 \mathrm{H})$ & $7.73(\mathrm{brs} 1 \mathrm{H})$ & $7.73(\mathrm{brs}, 1 \mathrm{H})$ \\
\hline $7.46(\mathrm{~d}, J=7.7 \mathrm{~Hz}, 1 \mathrm{H})$ & $7.47(\mathrm{~d} J=7.8 \mathrm{~Hz}, 1 \mathrm{H})$ & $7.47(\mathrm{~d}, J=7.8 \mathrm{~Hz}, 1 \mathrm{H})$ \\
\hline 729 (dt, $J=79 \mathrm{~Hz}, 1 \mathrm{H})$ & $731(\mathrm{~d}, J=7.8 \mathrm{~Hz}, 1 \mathrm{H})$ & $7.30(\mathrm{~d}, J=7.8 \mathrm{~Hz}, 1 \mathrm{H})$ \\
\hline $7.14-7.06(\mathrm{~m}, 2 \mathrm{H})$ & $7.16-7.11(\mathrm{~m}, 1 \mathrm{H})$ & $7.16-7.11(\mathrm{~m}, 1 \mathrm{H})$ \\
\hline & $7.11-7.06(\mathrm{~m}, 1 \mathrm{H})$ & $7.10-7.06(\mathrm{~m}, 1 \mathrm{H})$ \\
\hline $422\left(\mathrm{brs}_{1} 1 \mathrm{H}\right)$ & $423(\mathrm{brs} 1 \mathrm{H})$ & $4.23(\mathrm{brs}, 1 \mathrm{H})$ \\
\hline $381(5,3 \mathrm{H})$ & $382(5,3 \mathrm{H})$ & $3.81(\mathrm{~s}, 3 \mathrm{H})$ \\
\hline $3.33(\mathrm{brd}, J=11.3 \mathrm{~Hz}, 1 \mathrm{H})$ & 335 (brd $f=107 \mathrm{~Hz}, 1 \mathrm{H})$ & $3.35\left(\right.$ br d, $\left.J=11.2 \mathrm{~Hz}_{1} 1 \mathrm{H}\right)$ \\
\hline $307(\mathrm{~m}, 1 \mathrm{H})$ & $309(\mathrm{dd}, J=10.7,54 \mathrm{~Hz}, 1 \mathrm{H})$ & $3.09(\mathrm{dd}, J=11.2,4.9 \mathrm{~Hz}, 1 \mathrm{H})$ \\
\hline $302-2.93(\mathrm{~m}, 3 \mathrm{H})$ & $304-2.95(\mathrm{~m}, 1 \mathrm{H})$ & $3.03-295(\mathrm{~m}, 1 \mathrm{H})$ \\
\hline & $296(\mathrm{dd}, J=11.2,29 \mathrm{~Hz}, 1 \mathrm{H})$ & $2.96(\mathrm{dd} J=112,2.9 \mathrm{~Hz}, 1 \mathrm{H})$ \\
\hline & $291(\mathrm{brs}, 1 \mathrm{H})$ & $2.95-289(\mathrm{brs}, 1 \mathrm{H})$ \\
\hline $272(\mathrm{dd}, J=152,4.2 \mathrm{~Hz}, 1 \mathrm{H})$ & $2.75-2.69(\mathrm{~m}, 1 \mathrm{H})$ & $2.75-2.69(\mathrm{~m}, 1 \mathrm{H})$ \\
\hline $262(\mathrm{td}, J=113,43 \mathrm{~Hz}, 1 \mathrm{H})$ & $\begin{array}{l}2.63(\mathrm{ddd}, J=11.2,11.2,4.4 \\
\mathrm{Hz}, 1 \mathrm{H})\end{array}$ & $\begin{array}{l}2.63(\mathrm{ddd}, J=11.2,112,4.4 \mathrm{~Hz}, 1 \\
\mathrm{H})\end{array}$ \\
\hline $234(\mathrm{dd}, J=115,1.9 \mathrm{~Hz}, 1 \mathrm{H})$ & $236(\mathrm{dd}, J=11.7,24 \mathrm{~Hz}, 1 \mathrm{H})$ & $2.36(\mathrm{dd}, J=112,2.0,1 \mathrm{H})$ \\
\hline $227-2.23(\mathrm{~m}, 1 \mathrm{H})$ & $229-2.23(\mathrm{~m}, 1 \mathrm{H})$ & $2.292223(\mathrm{~m}, 1 \mathrm{H})$ \\
\hline $204-1.98(\mathrm{~m}, 3 \mathrm{H})$ & $2008-1.96(\mathrm{~m}, 3 \mathrm{H})$ & $2.07-196(\mathrm{~m}, 3 \mathrm{H})$ \\
\hline $158(\mathrm{~m}, 3 \mathrm{H})$ & $1.64-1.52(\mathrm{~mm} 3 \mathrm{H})$ & $1.65-1.51(\mathrm{~m}, 3 \mathrm{H})$ \\
\hline $1.43(\mathrm{~m}, 1 \mathrm{H})$ & $1.47-1.42(\mathrm{~m}, 1 \mathrm{H})$ & $1.46-1.42(\mathrm{~m}, 1 \mathrm{H})$ \\
\hline $137(\mathrm{q}, J=117 \mathrm{~Hz} 1 \mathrm{H})$ & $\begin{array}{l}1.38(\mathrm{ddd}, J=11.2,11.2,11.2 \\
\mathrm{Hz}, 1 \mathrm{H})\end{array}$ & $\begin{array}{l}1.38(\text { ddd, } J=11.7,11.7,11.7 \mathrm{~Hz} \\
1 \mathrm{H})\end{array}$ \\
\hline
\end{tabular}

s) Aube, I. Chodh, S.: Tanol, M. J.Am. Cham. Soo. 1994, 116,9009-9018. b) Duta were obtained by mes:ring the 'F NMR spectum of natural 1 


\begin{tabular}{|c|c|c|}
\hline \multicolumn{3}{|c|}{ Comparison of ${ }^{15}$ C NMR Spectral Data for (+)-Yohimbine (1) } \\
\hline Aube $^{4}$ & This Work & Natural \\
\hline 175.6 & 175.6 & 175.6 \\
\hline 135.9 & 136.0 & 135.9 \\
\hline 134.5 & 134.3 & 134.5 \\
\hline 127.4 & 127.4 & 127.4 \\
\hline 121.3 & 121.4 & 121.3 \\
\hline 119.4 & 119.4 & 119.3 \\
\hline 118.1 & 118.1 & 118.1 \\
\hline 110.7 & 110.7 & 110.7 \\
\hline 108.2 & 108.3 & 108.2 \\
\hline 66.9 & 66.9 & 66.9 \\
\hline 61.3 & 61.3 & 61.3 \\
\hline 59.8 & 59.9 & 59.8 \\
\hline 52.9 & 52.9 & 52.8 \\
\hline 52.3 & 52.3 & 52.3 \\
\hline 51.9 & 52.0 & 51.9 \\
\hline 40.7 & 40.7 & 40.7 \\
\hline 36.7 & 36.7 & 36.7 \\
\hline 34.3 & 34.3 & 34.3 \\
\hline 31.4 & 31.4 & 31.5 \\
\hline 23.3 & 23.3 & 23.3 \\
\hline 21.7 & 21.7 & 21.7 \\
\hline
\end{tabular}

a) Aubé, J.; Ghosh, S.; Tanol, M. J. Am. Chem. Soc. 1994, 116, 9009-9018. b) Data were obtained by recording the ${ }^{13} \mathrm{C}$ NMR spectrum of $\mathbf{1}$ obtained commercially from Aldrich. 

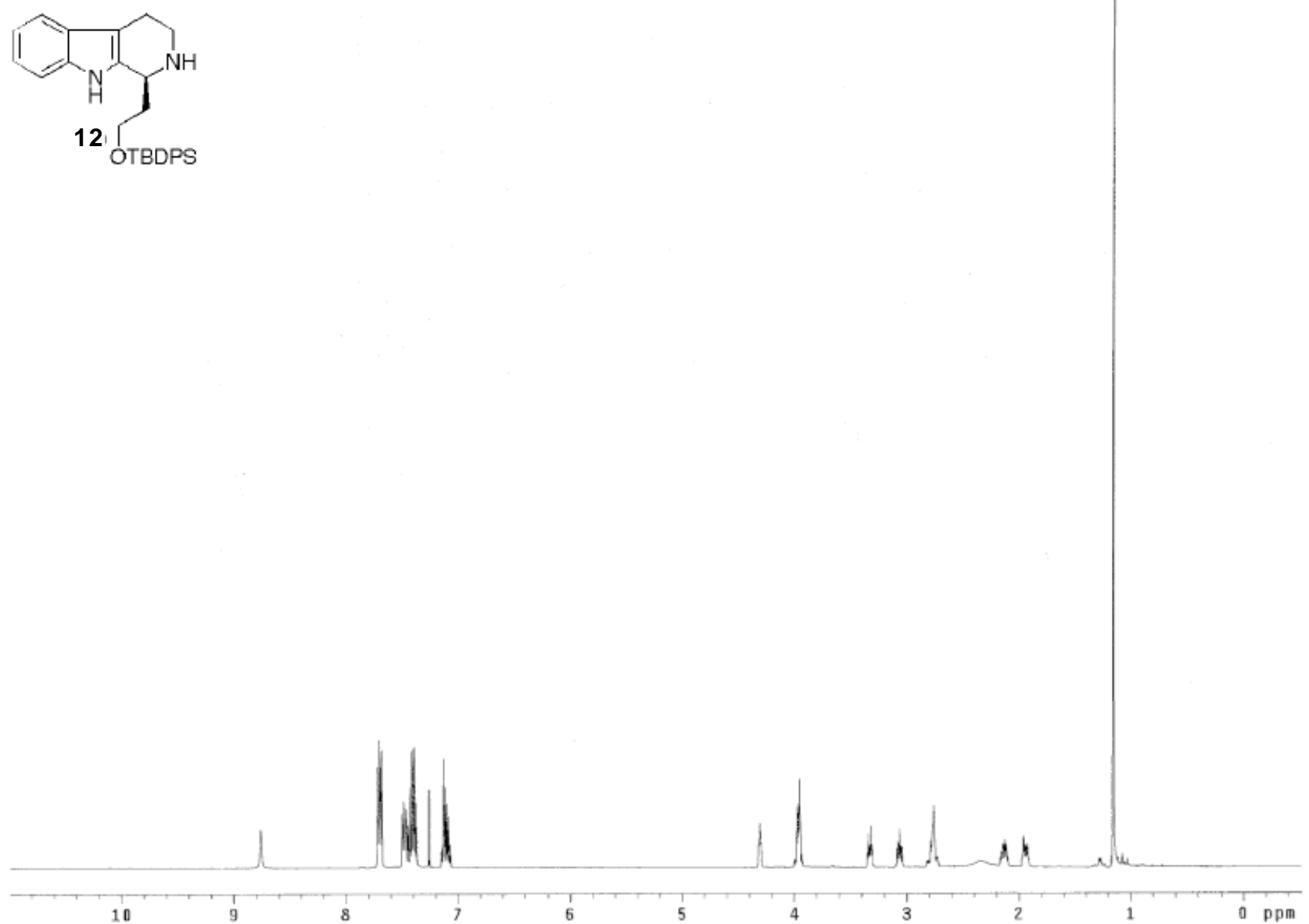

S-15 


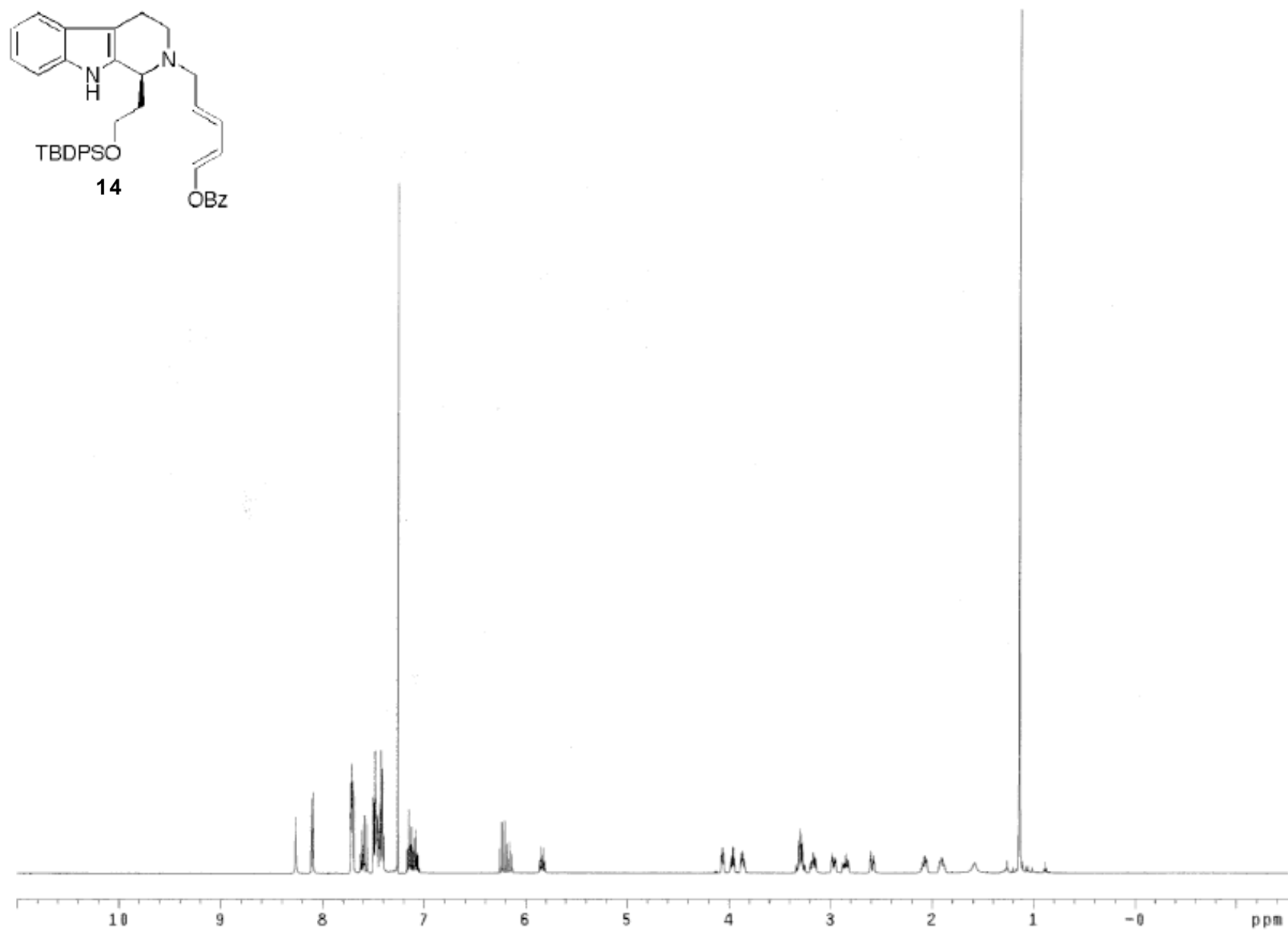

S-16 


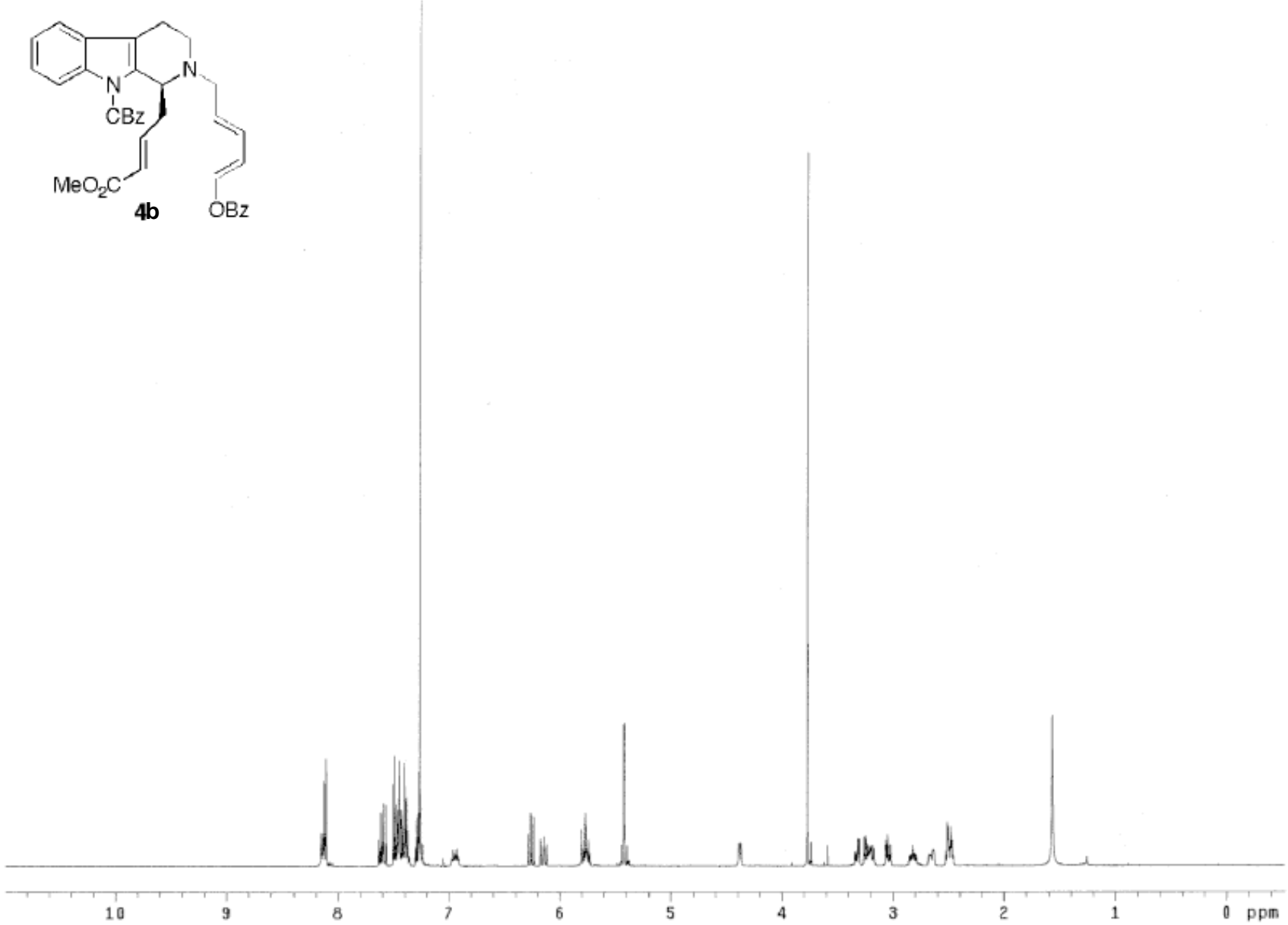

S-17 


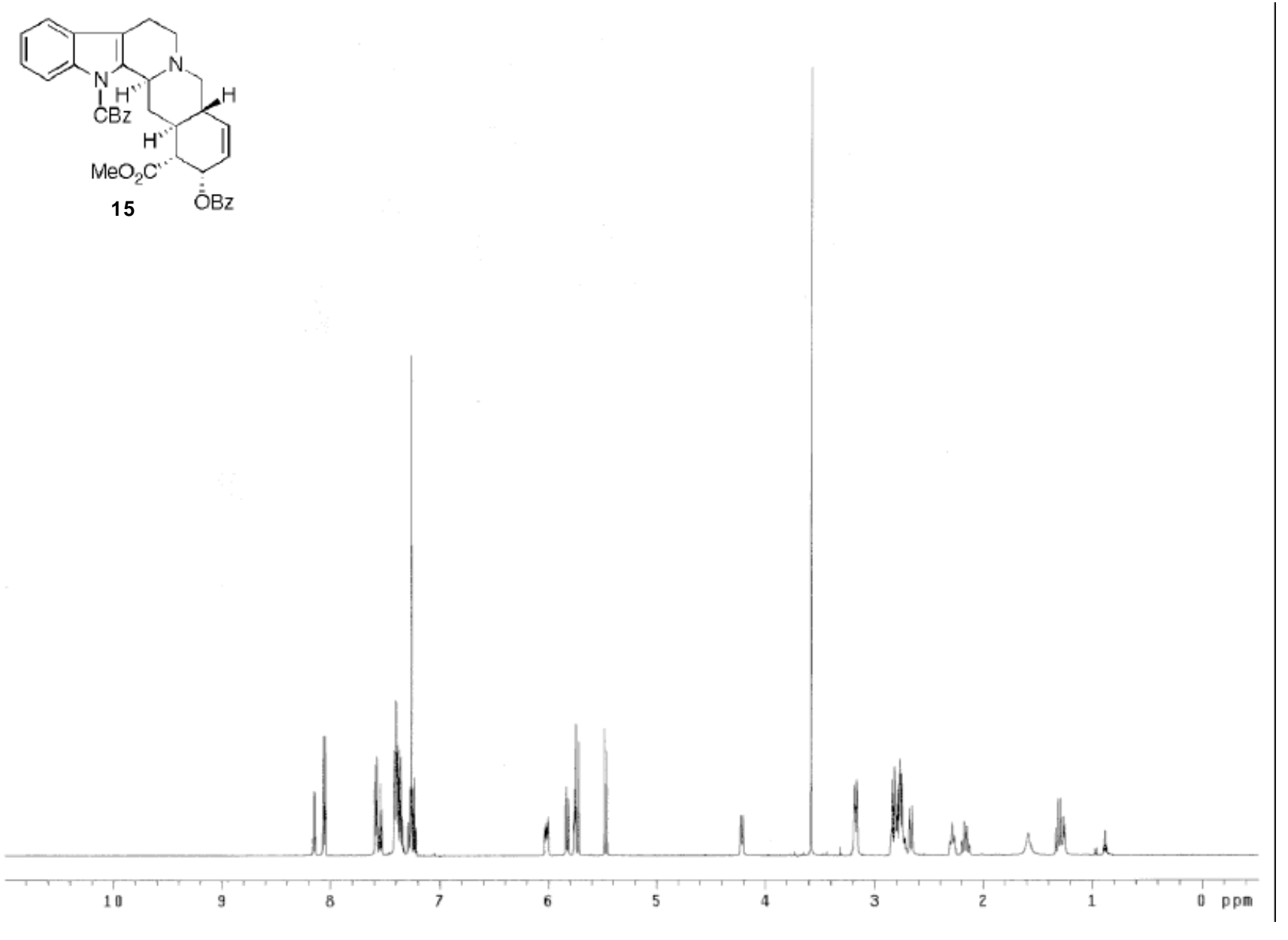

S-18 


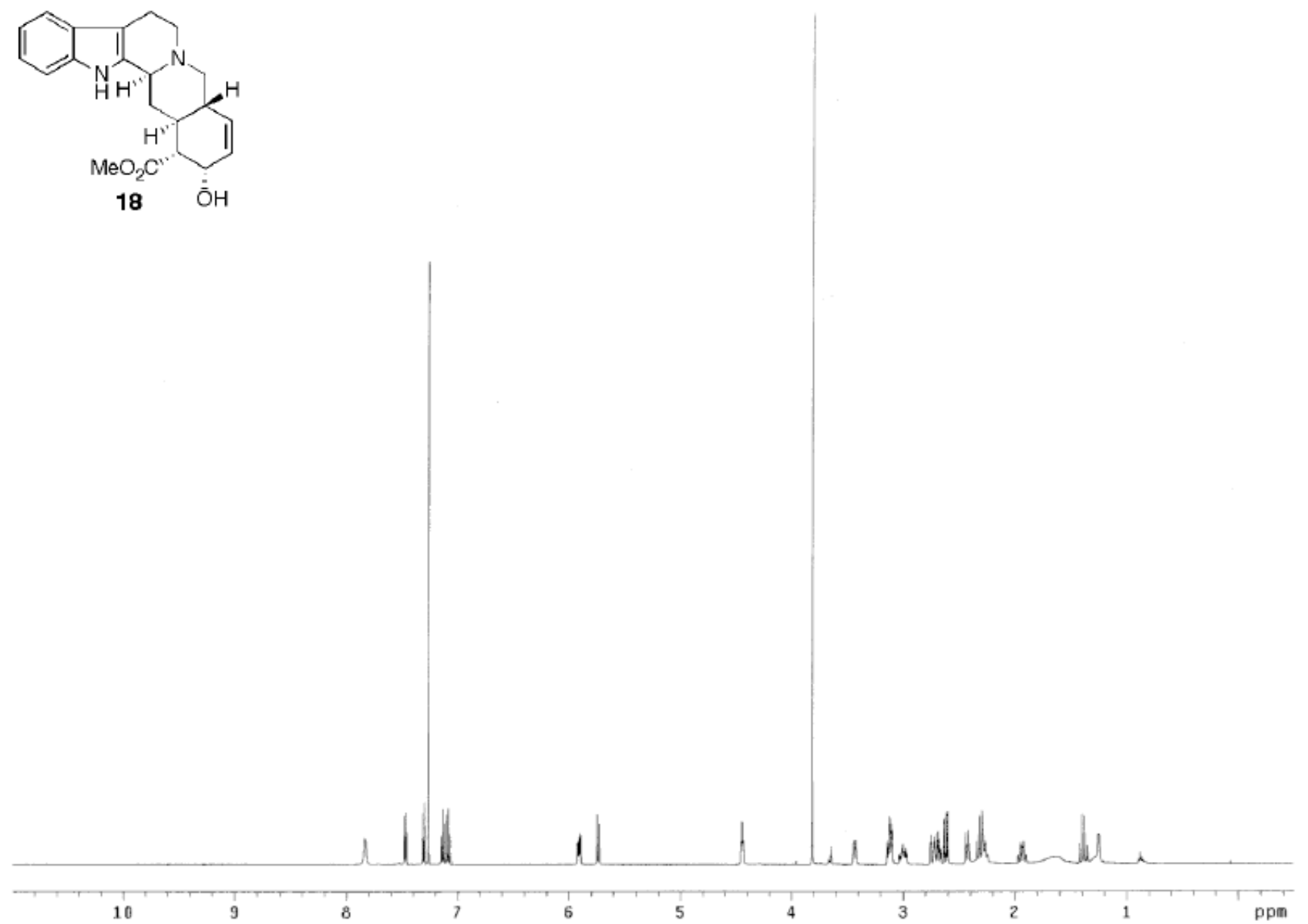

S-19 


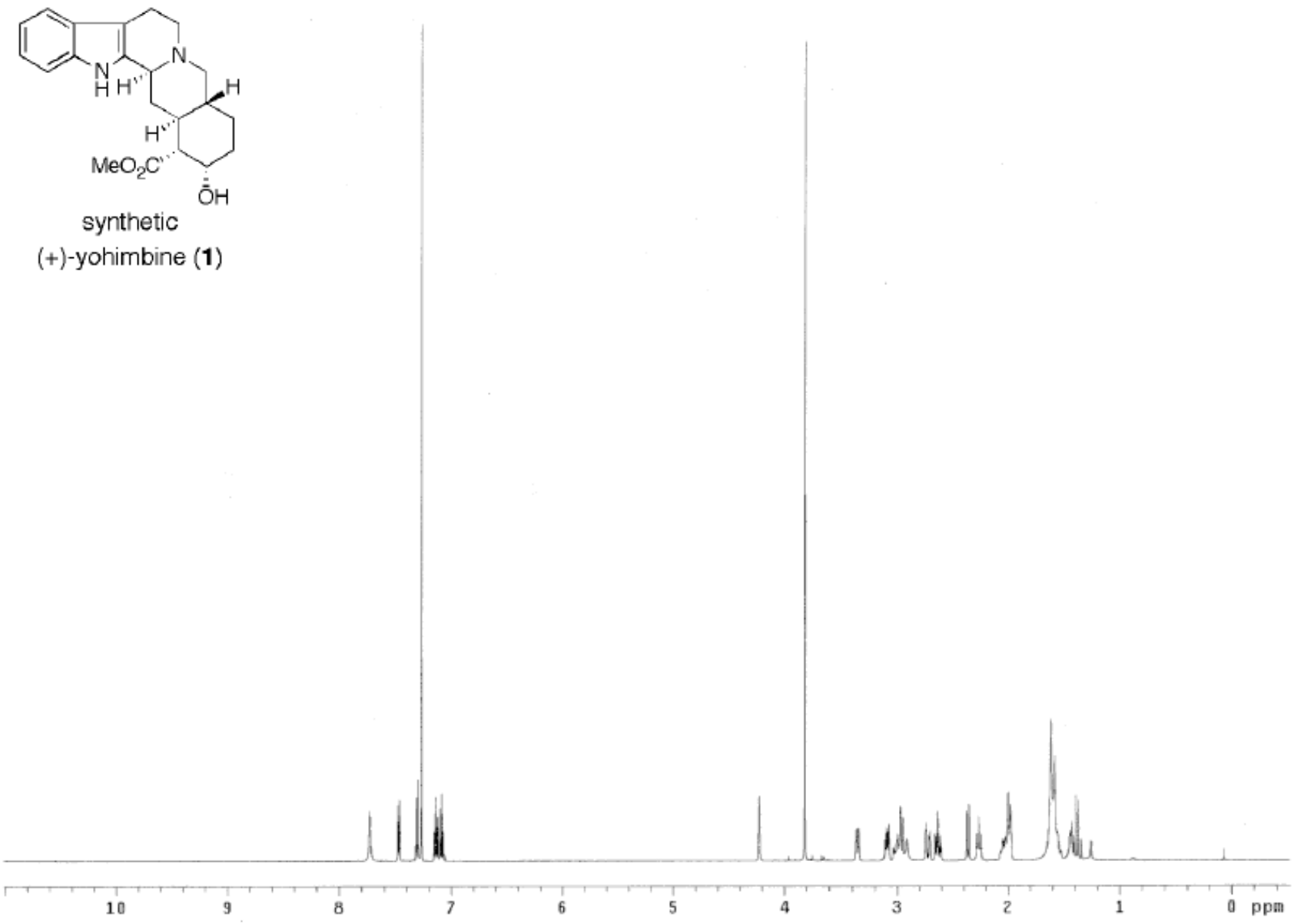




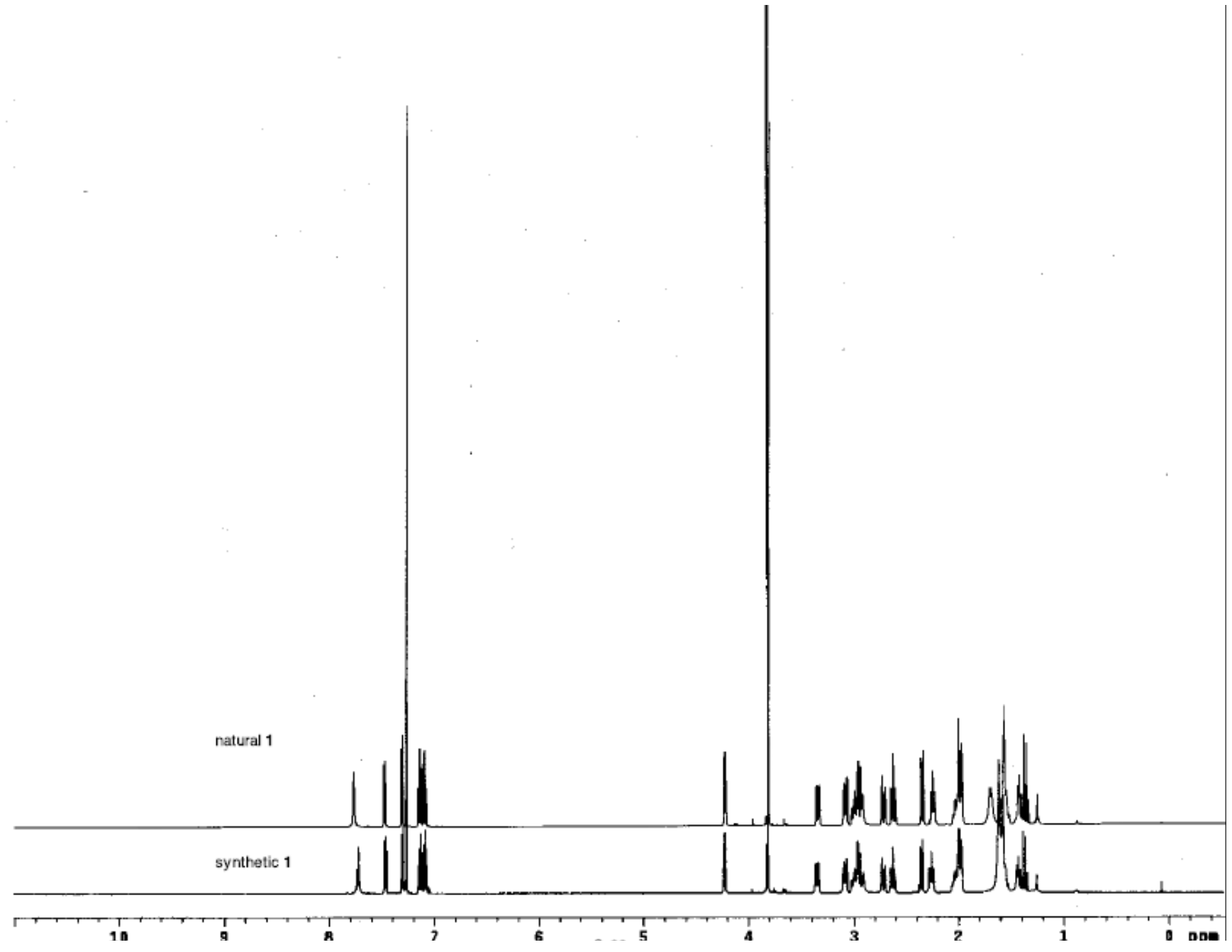




\section{Calculations}

A. Methods. Calculations were performed using the Gaussian 98 program $^{5}$ with the $\mathrm{B}^{2} \mathrm{LYP}^{6}$ or MP2 $^{7}$ methods, as specified. The 6-31G(d), 6-31+G(d), and 6-311+G(d,p) basis sets of Pople and coworkers were used, as specified. ${ }^{8}$ All B3LYP/6-31G(d) transition structures were shown to be legitimate firstorder saddle points by the existence of a single imaginary frequency. Single-point energy calculations at the B3LYP/6-31+G(d)// B3LYP/6-31G(d) and B3LYP/6-311+G(d,p)//B3LYP/6-31G(d) levels employed the keyword $\mathrm{SCF}=$ Tight. Unscaled zero-point vibrational energy corrections at the B3LYP/6-31G(d) level are included where specified. The following units are used: relative energy, kcal/mol; absolute energy, Hartree; distance, Å.

B. Model Study. A model study was conducted to evaluate the effect of level of theory and basis set on the geometries (i.e. length of forming $\mathrm{C}-\mathrm{C}$ bonds) and relative energies of Diels-Alder transition structures.

(5) Gaussian 98, Revision A.11.3, Frisch, M. J.; Trucks, G. W.; Schlegel, H. B.; Scuseria, G. E.; Robb, M. A.; Cheeseman, J. R.; Zakrzewski, V. G.; Montgomery, Jr., J. A.; Stratmann, R. E.; Burant, J. C.; Dapprich, S.; Millam, J. M.; Daniels, A. D.; Kudin, K. N.; Strain, M. C.; Farkas, O.; Tomasi, J.; Barone, V.; Cossi, M.; Cammi, R.; Mennuci, B.; Pomelli, C.; Adamo, C.; Clifford, S.; Ochterski, J.; Petersson, G. A.; Ayala, P. Y.; Cui, Q.; Morokuma, K.; Rega, N.; Salvador, P.; Dannenberg, J. J.; Malick, D. K.; Rabuck, A. D.; Raghavachari, K.; Foresman, J. B.; Cioslowski, J.; Ortiz, J. V.; Baboul, A. G.; Stefanov, B. B.; Liu, G.; Liashenko, A.; Piskorz, P.; Komaromi, I.; Gomperts, R.; Martin, R. L.; Fox, D. J.; Keith, T.; Al-Laham, M. A.; Peng, C. Y.; Nanayakkara, A.; Challacombe, M.; Gill, P. M. W.; Johnson, B.; Chen, W.; Wong, M. W.; Gonzalez, C.; Head-Gordon, M; Replogle, E. S.; Pople, J. A.; Gaussian, Inc., Pittsburgh PA, 2002.

(6) B3LYP = Becke-3-Lee-Yang-Parr density functional theory. (a) Becke, A. D. J. Chem. Phys. 1993, 98, 1372-1377. (b) Lee, C.; Yang, W.; Parr, R. G. Phys. Rev. B 1988, 37, 785-789.

(7) Møller, C.; Plesset, M. S. Phys. Rev. 1934, 46, 618-622.

(8) (a) Ditchfield, R.; Hehre, W. J.; Pople, J. A. J. Chem. Phys. 1971, 54, 724-728. (b) Hehre, W. J.; Ditchfield, R.; Pople, J. A. J. Chem. Phys. 1972, 56, 2257-2261. (c) Hariharan, P. C.; Pople, J. A. Theor. Chim. Acta. 1973, 28, 213-223. 
Endo transition structures:

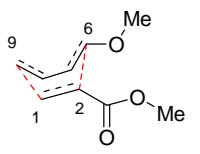

Geometry

B3LYP:

6-31G(d)

6-311G+G(d,p)

MP2:

$6-31+G(d)$

Relative Energy

B3LYP:

6-31G(d)

6-31G+G(d)

$6-311 G+G(d, p)$

$6-31 G+G(d) / / 6-31 G(d)$

$6-311 G+G(d, p) / / 6-31 G(d)$

6-311G+G(d,p)/

6-31G(d)/6-31G(d)

MP2:

6-31G(d)

$6-31+G(d)$
$6-31+G(d) / / 6-31 G(d)$

Exo transition structures:

Geometry

B3LYP:

6-31G(d)

6-31G+G(d)

MP2:

6-31G(d)

Relative Energy

B3LYP:

6-31G(d)

$6-311 G+G(d, p)$

6-31G+G(d)//6-31G(d)

6-311G+G(d,p)//6-31G(d)

MP2//B3LYP:

6-31G(d)//6-31G(d)

MP2:

6-31G(d)

$6-31+G(d) / / 6-31 G(d)$

$2.139 / 2.570$

$2.134 / 2.579$

0.0 (def)

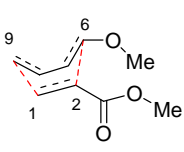

C1-C9/C2-C6

$1.997 / 2.699$

$1.973 / 2.747$

$1.963 / 2.712$

$2.112 / 2.506$

$2.146 / 2562$

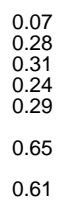

0.83
0.79
1.04
0.79
1.01
0.62
0.45

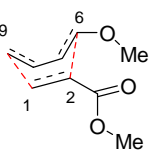

C1-C9/C2-C6

$2.000 / 2.669$

$1.983 / 2.703$

$1.972 / 2.672$

$2.105 / 2.504$

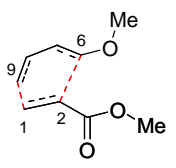

C1-C9/C2-C6

$1.989 / 2.716$

$1.969 / 2.760$

$1.961 / 2.720$

$2.113 / 2.530$
$2.112 / 2.531$

Energies are electronic energies and do not include zero-point vibrational energy corrections.

\section{Relative energies and geometries of IMDA transition structures using substrate $\underline{4 a}$.}


Endo, C(3)-C(15) cis:

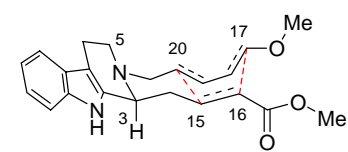

Geometry, B: 2.040/2.657

Geometry, M: 2.171/2.576

B/6-31G(d): 2.91

$B / 6-31+G(d): 2.96$

$B / 6-311+G(d, p): 2.58$

M/6-31G(d): 2.18

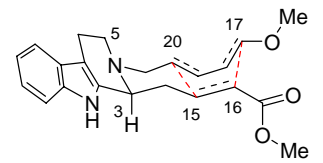

Geometry, B: 2.055/2.639

$B / 6-31 G(d): 3.47$

$B / 6-31+G(d): 3.40$

$B / 6-311+G(d, p): 3.27$

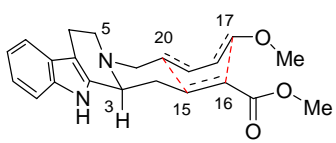

Geometry, B: 2.004/2.642

B/6-31G(d): 4.42

$B / 6-31+G(d): 4.31$

$\mathrm{B} / 6-311+\mathrm{G}(\mathrm{d}, \mathrm{p}): 4.21$

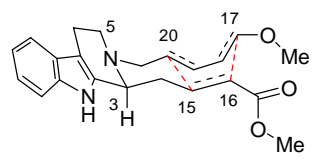

Geometry, B: 2.010/2.614

B/6-31G(d): 5.14

$B / 6-31+G(d): 5.32$

B/6-311+G(d,p): 5.18

Endo, C-ring chair, C(3)-C(15) trans:

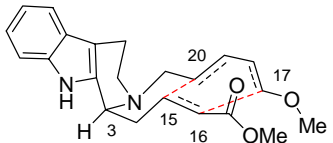

Geometry, B: 2.043/2.670

B/6-31G(d): 3.78

$\mathrm{B} / 6-311+\mathrm{G}(\mathrm{d}, \mathrm{p}): 3.18$

$\mathrm{M} / 6-31 \mathrm{G}(\mathrm{d}): 0.77$

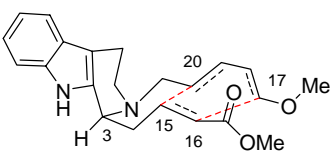

Geometry, B: 2.008/2.655

B/6-31G(d): 5.11

$B / 6-311+G(d, p): 4.71$

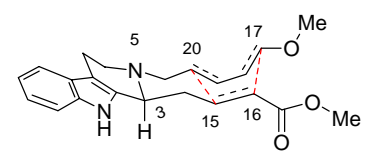

Geometry, B: $2.049 / 2.675$

Geometry, M: 2.174/2.609

B/6-31G(d): 0.71

$B / 6-31+G(d): 0.42$

$B / 6-311+G(d, p): 0.06$

M/6-31G(d): 0.46

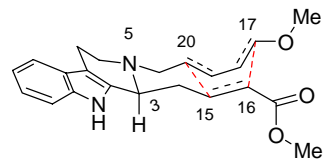

Geometry, B: 2.055/2.670

B/6-31G(d): 1.14

$B / 6-31+G(d): 0.94$

$B / 6-311+G(d, p): 0.66$

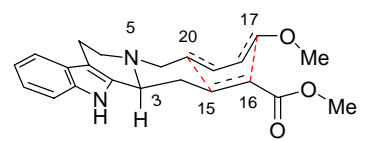

Geometry, B: 2.014/2.662

$B / 6-31 G(d): 2.15$

$B / 6-31+G(d): 1.76$

$B / 6-311+G(d, p): 1.63$

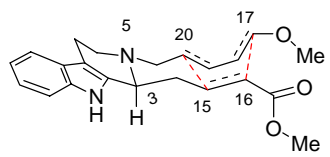

Geometry, B: 2.015/2.637

B/6-31G(d): 2.89

$B / 6-31+G(d): 2.86$

$B / 6-311+G(d, p): 2.62$

Endo, C-ring boat, C(3)-C(15) trans:

(1)

Geometry, B: 2.014/2.617

B/6-31G(d): 5.04

$B / 6-311+G(d, p): 4.55$

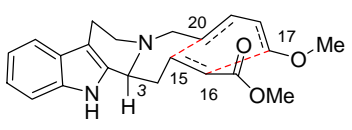

Geometry, B: 1.980/2.604

$B / 6-31 G(d): 6.14$

$B / 6-311+G(d, p): 5.84$

Exo, C(3)-C(15) cis:

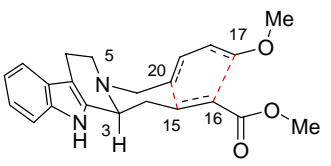

Geometry, B: 2.014/2.612 Geometry, M: 2.157/2.518

B/6-31G(d): 4.86

$B / 6-31+G(d): 5.00$

$B / 6-311+G(d, p): 4.91$

M/6-31G(d): 4.18

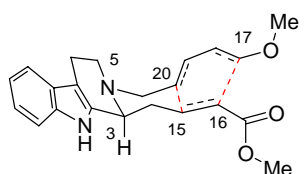

Geometry, B: 2.039/2.570

$B / 6-31 G(d): 6.55$

$B / 6-31+G(d): 6.48$

$B / 6-311+G(d, p): 6.39$

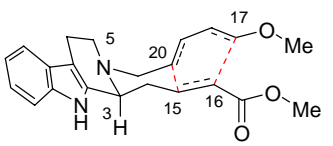

Geometry, B: 2.001/2.738 Geometry, M: 2.158/2.613

$B / 6-31 G(d): 4.48$$$
B / 6-311+G(d, p): 4.80
$$

$\mathrm{B} / 6-311+\mathrm{G}(\mathrm{d}, \mathrm{p}): 4.80$
$\mathrm{M} / 6-31 \mathrm{G}(\mathrm{d}): 4.97$

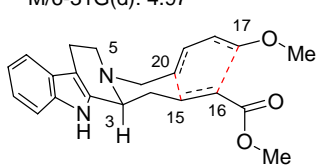

Geometry, B: 2.006/2.703

$B / 6-31 G(d): 5.20$

$B / 6-31+G(d): 5.72$

Exo, C-ring chair, $\mathrm{C}(3)-\mathrm{C}(15)$ trans:

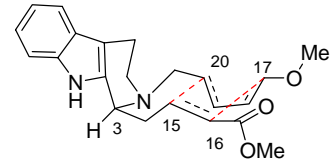

Geometry, B: 1.991/2.797

B/6-31G(d): 2.88

p): 3.04

M/6-31G(d): 0.72

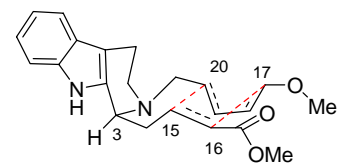

Geometry, B: 1.994/2.679

$B / 6-31 G(d): 3.68$

$B / 6-311+G(d, p): 3.63$

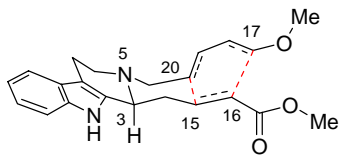

Geometry, B: $2.001 / 2.683$ Geometry, M: 2.142/2.590

B/6-31G(d): 0.68

$B / 6-31+G(d): 0.77$

$B / 6-311+G(d, p): 0.49$

M/6-31G(d): 0.0 (def)

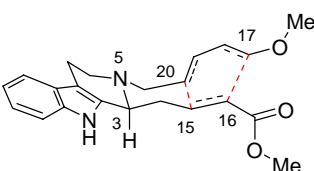

Geometry, B: 2.019/2.653

B/6-31G(d): 2.44

$B / 6-31+G(d): 2.39$

$B / 6-311+G(d, p): 2.02$

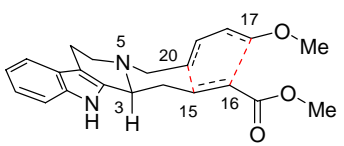

Geometry, B: 1.999/2.808 Geometry, M: 2.147/2.684

B/6-31G(d): 0.0 (def)

$B / 6-31+G(d): 0.0$ (def)

$B / 6-311+G(d, p): 0.0$ (def)

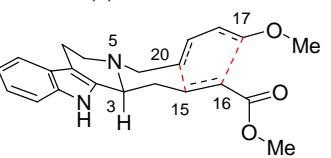

Geometry, B: 2.005/2.768

B/6-31G(d): 0.71

$B / 6-31+G(d): 0.67$

$B / 6-311+G(d, p): 0.54$

Exo, C-ring boat, $\mathrm{C}(3)-\mathrm{C}(15)$ trans:

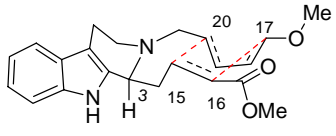

Geometry, B: 1.970/2.703

$B / 6-31 G(d): 5.02$

$B / 6-311+G(d, p): 5.10$

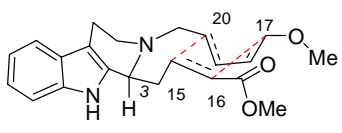

Geometry, B: 1.985/2.579

B/6-31G(d): 5.79

$B / 6-311+G(d, p): 5.72$ 


\section{Relative energies and geometries of IMDA transition structures using substrate $\underline{5}$.}

Endo, C(3)-C(15) cis:
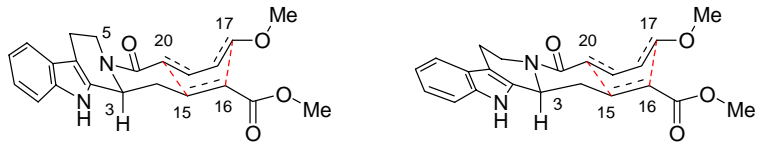

Geometry, B: 2.049/2.654

B/6-31G(d): 6.88

B/6-311+G(d,p): 6.38

Geometry, B: 2.047/2.634

B/6-31G(d): 0.98

$B / 6-311+G(d, p): 0.58$

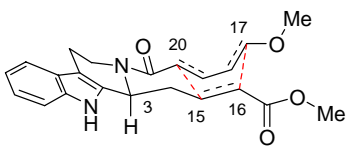

Geometry, B: 2.012/2.618

$B / 6-31 G(d): 2.13$

Exo, C(3)-C(15) cis:

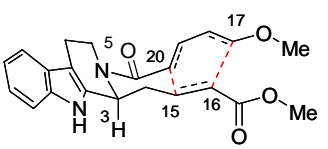

Geometry, B: 2.003/2.654

B/6-31G(d): 7.55

$B / 6-311+G(d, p): 6.83$

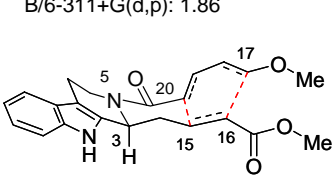

Geometry, B: 1.993/2.803

B/6-31G(d): 1.72

$B / 6-311+G(d, p): 0.91$

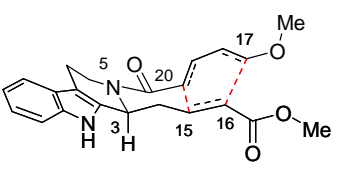

Geometry, B: 2.003/2.675

B/6-31G(d): 2.50 $B / 6-311+G(d, p): 1.78$
Endo, C-ring chair, C(3)-C(15) trans:

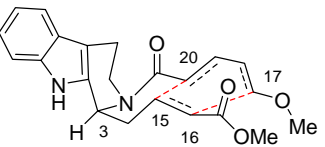

Geometry, B: $2.039 / 2.618$

$\mathrm{B} / 6-31 \mathrm{G}(\mathrm{d}): 1.70$

$B / 6-311+G(d, p): 1.28$

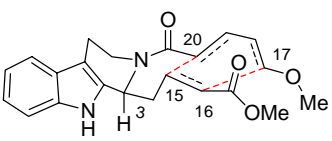

Geometry, B: 2.022/2.584

B/6-31G(d): 0.25

$B / 6-311+G(d, p): 0.25$

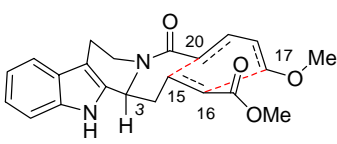

Geometry, B: $1.987 / 2.568$

$B / 6-31 G(d): 1.41$

$B / 6-311+G(d, p): 1.54$

Exo, C-ring chair, $\mathrm{C}(3)-\mathrm{C}(15)$ trans:

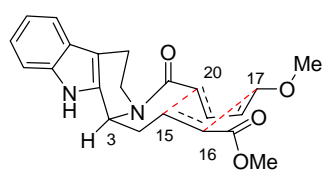

Geometry, B: 1.986/2.779

B/6-31G(d): 2.60

$B / 6-311+G(d, p): 2.14$
Endo, C-ring boat, C(3)-C(15) trans:

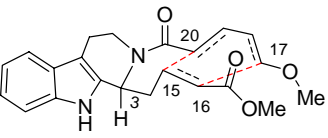

Geometry, B: 2.034/2.524

B/6-31G(d): 0.0 (def.) $B / 6-311+G(d, p): 0.0$ (def.)

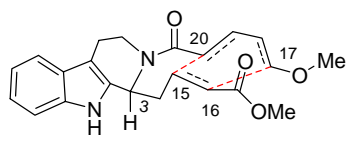

Geometry, B: 1.998/2.506

B/6-31G(d): 1.32

$B / 6-311+G(d, p): 1.49$

Exo, C-ring boat, $\mathrm{C}(3)-\mathrm{C}(15)$ trans:

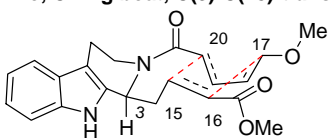

Geometry, B: $1.963 / 2.788$

B/6-31G(d): 2.80

$B / 6-311+G(d, p): 2.56$

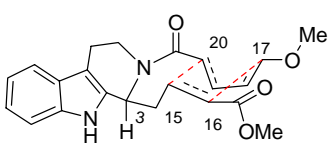

Geometry, B: 1.959/2.719

B/6-31G(d): 3.24 $B / 6-311+G(d, p): 3.15$ 


\section{E. Relative energies and geometries of IMDA transition structures using substrate $\underline{4 c}$.}

Endo, C(3)-C(15) cis:

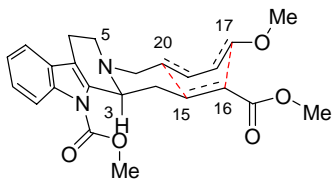

Geometry, B: 2.043/2.649

B/6-31G(d): 0.78

$B / 6-311+G(d, p): 0.82$

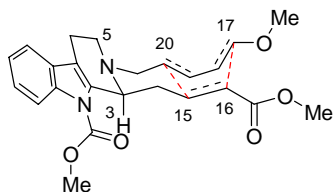

Geometry, B: $2.040 / 2.649$

$B / 6-31 G(d): 0.88$

$B / 6-311+G(d, p): 0.91$

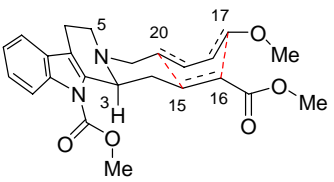

Geometry, B: 2.007/2.639

$B / 6-31 G(d): 2.29$

$B / 6-311+G(d, p): 2.24$

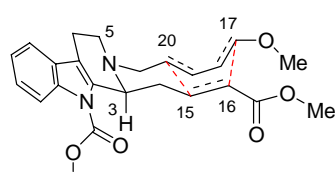

$\mathrm{Me}$

Geometry, B: 2.004/2.640

$\mathrm{B} / 6-31 \mathrm{G}(\mathrm{d}): 2.34$

$B / 6-311+G(d, p): 2.53$

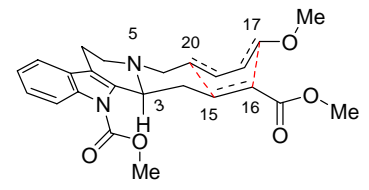

Geometry, B: 2.045/2.664

B/6-31G(d): 0.15

$B / 6-311+G(d, p): 0.0$ (def)

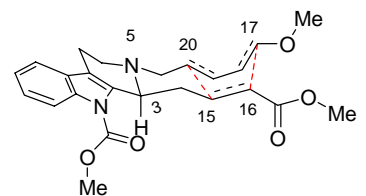

Geometry, B: 2.044/2.669

B/6-31G(d): 0.54

$B / 6-311+G(d, p): 0.31$

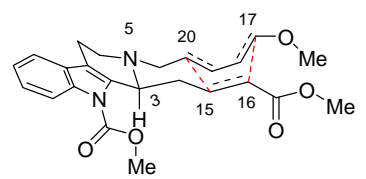

Geometry, B: 2.013/2.652

B/6-31G(d): 1.70

$B / 6-311+G(d, p): 1.66$

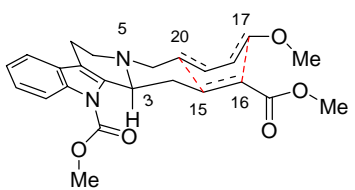

Geometry, B: 2.011/2.660

B/6-31G(d): 1.98

$B / 6-311+G(d, p): 1.89$
Exo, C(3)-C(15) cis

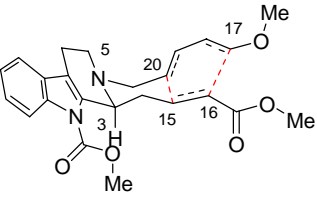

Geometry, B: 2.024/2.593

B/6-31G(d): 3.24

$\mathrm{B} / 6-311+\mathrm{G}(\mathrm{d}, \mathrm{p}): 4.29$
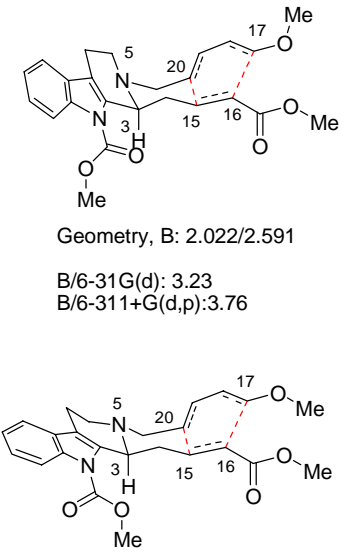

Geometry, B: 1.996/2.804

$B / 6-31 G(d): 0.01$

$B / 6-311+G(d, p): 0.35$

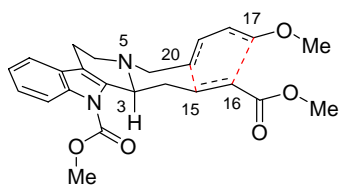

$B / 6-311+G(d, p): 0.41$

Exo, C-ring chair, $\mathrm{C}(3)-\mathrm{C}(15)$ trans:

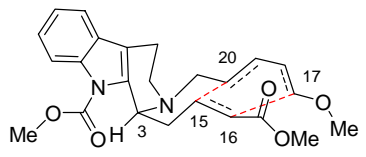

Geometry, B: 2.036/2.688

B/6-31G(d): 5.33

$B / 6-311+G(d, p): 4.95$
Me

Geometry, B: 2.022/2.591

$B / 6-31 G(d): 3.23$

$B / 6-311+G(d, p): 3.76$

Geometry, B: 1.997/2.796

$B / 6-31 G(d): 0.0$ (def)

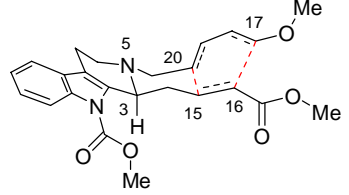

Geometry, B: 2.003/2.673

B/6-31G(d): 0.58

$B / 6-311+G(d, p): 0.77$

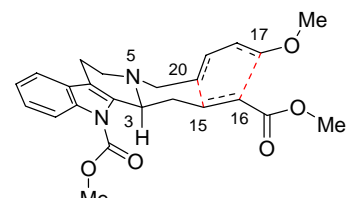

$\mathrm{Me}$

Geometry, B: $2.000 / 2.678$

B/6-31G(d): 0.71

$B / 6-311+G(d, p): 0.98$

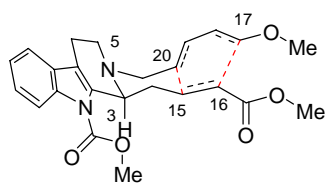

Geometry, B: 2.003/2.731

B/6-31G(d): 2.96

$B / 6-311+G(d, p): 3.41$

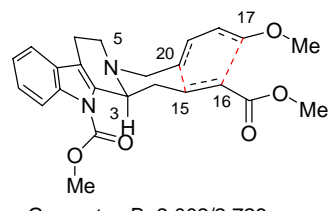

Geometry, B: 2.002/2.726

B/6-31G(d): 2.84

$B / 6-311+G(d, p): 3.46$

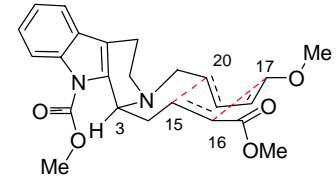

Geometry, B: 1.990/2.823

B/6-31G(d): 5.25

$B / 6-311+G(d, p): 5.42$

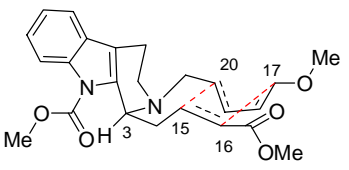

Geometry, B: 1.985/2.823

B/6-31G(d): 4.79

$B / 6-311+G(d, p): 5.16$

Geometry = C15-C20/C16-C17; all geometries obtained using the 6-31G(d) basis set. B = B3LYP, M = MP2.

Energies are electronic energies and include unscaled B3LYP/6-31G(d) zero-point vibrational energy corrections. 


\section{F. Relative energies and geometries of IMDA transition structures using substrate $\underline{4} \cdot \mathbf{B H}_{3}$.}

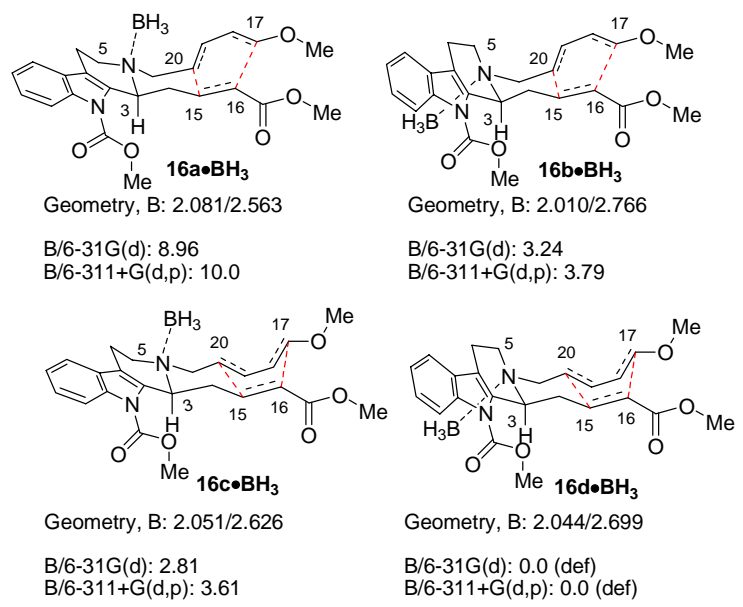

G. Torsional energy profile for the rotation of a carbamate $\mathrm{C}=\mathbf{O}$ relative to the indole aromatic plane. Two stationary points (I and III) were located at the B3LYP/6-31G(d) level and shown to be local minima by the existence of no imaginary frequencies. The C-N-C-O dihedral angle shown in red was then fixed and varied in $20^{\circ}$ increments and all other coordinates fully relaxed. The relative energies of the resulting structures at the B3LYP/6-31G(d)//B3LYP/6-31G(d) $(\bullet)$ and B3LYP/6-311+G(d,p)//B3LYP/6-31G(d) ( $\mathbf{\nabla})$ are plotted below. The C-N-C-O dihedral angles of local minima I and III are $0.01^{\circ}$ and $179.97^{\circ}$.
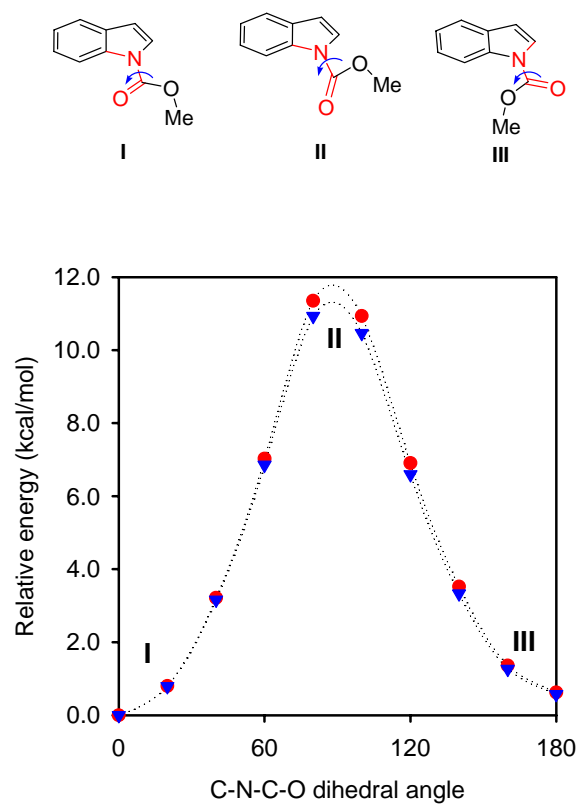

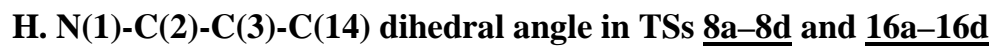

\begin{tabular}{cccc}
\hline TS & $\begin{array}{c}\mathrm{N}(1)-\mathrm{C}(2)-\mathrm{C}(3)-\mathrm{C}(14) \\
\text { dihedral angle }\end{array}$ & $\mathrm{TS}$ & $\begin{array}{c}\mathrm{N}(1)-\mathrm{C}(2)-\mathrm{C}(3)-\mathrm{C}(14) \\
\text { dihedral angle }\end{array}$ \\
\hline $\mathbf{8 a}$ & 45.3 & $\mathbf{1 6 a}$ & 51.9 \\
$\mathbf{8 b}$ & 59.9 & $\mathbf{1 6 b}$ & 65.6 \\
$\mathbf{8 c}$ & 47.6 & $\mathbf{1 6 c}$ & 54.8 \\
$\mathbf{8 d}$ & 64.8 & $\mathbf{1 6 d}$ & 69.2 \\
\hline
\end{tabular}


I. Complete geometries and absolute energies of transition structures depicted in Figures 1-3.

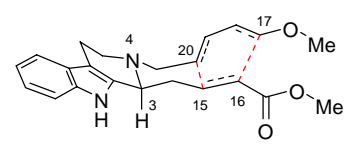

8a: $0.0 \mathrm{kcal} / \mathrm{mol}$

E (B3LYP/6-31G(d)): -1189.082081

E (B3LYP/6-311+G(d,p)//B3LYP/6-31G(d)): -1189.402627 ZPE (B3LYP/6-31G(d)): 0.446225

Imaginary frequencies: 1

\begin{tabular}{lrrr}
$\mathrm{C}$ & 4.57482600 & 0.01296600 & 0.22966800 \\
$\mathrm{C}$ & 4.18762600 & 1.38339800 & 0.20993900 \\
$\mathrm{C}$ & 5.06654900 & 2.41199900 & 0.55979100 \\
$\mathrm{C}$ & 6.35794200 & 2.05538700 & 0.93521400 \\
$\mathrm{C}$ & 6.76418300 & 0.70609800 & 0.96044300 \\
$\mathrm{C}$ & 5.88671300 & -0.31411200 & 0.61213700 \\
$\mathrm{C}$ & 3.42729400 & -0.74758700 & -0.18830400 \\
$\mathrm{C}$ & 2.41541900 & 0.14352500 & -0.43026300 \\
$\mathrm{H}$ & 4.75484600 & 3.45339300 & 0.53975600 \\
$\mathrm{H}$ & 7.06536400 & 2.83204300 & 1.21292600 \\
$\mathrm{H}$ & 7.78063600 & 0.46295200 & 1.25817700 \\
$\mathrm{H}$ & 6.21054100 & -1.35166800 & 0.63653600 \\
$\mathrm{C}$ & 1.03036000 & -0.19998600 & -0.90459200 \\
$\mathrm{C}$ & -0.05300000 & 0.58550500 & -0.13248600 \\
$\mathrm{H}$ & 0.93847000 & 0.10033500 & -1.97350100 \\
$\mathrm{C}$ & -0.42032800 & -2.08449700 & -1.38865200 \\
$\mathrm{H}$ & 0.01249900 & 0.34970200 & 0.93527700 \\
$\mathrm{H}$ & 0.17046900 & 1.65782800 & -0.23388600 \\
$\mathrm{H}$ & -0.40788000 & -3.17960000 & -1.45598300 \\
$\mathrm{H}$ & -0.53458700 & -1.71030200 & -2.42533200 \\
$\mathrm{~N}$ & 0.84357800 & -1.65770800 & -0.78941800 \\
$\mathrm{C}$ & 3.22579900 & -2.21707800 & -0.39698500 \\
$\mathrm{C}$ & 1.99468500 & -2.42169200 & -1.29032300 \\
$\mathrm{H}$ & 4.10048400 & -2.67665600 & -0.87648200 \\
$\mathrm{H}$ & 1.71127600 & -3.47948900 & -1.30069800 \\
$\mathrm{H}$ & 2.24633500 & -2.14089100 & -2.33172700 \\
$\mathrm{H}$ & 3.07635500 & -2.74031900 & 0.55838300 \\
$\mathrm{~N}$ & 2.86774300 & 1.43329600 & -0.19889400 \\
$\mathrm{H}$ & 2.32003400 & 2.27391500 & -0.29214700 \\
$\mathrm{C}$ & -1.46116700 & 0.35515200 & -0.65142200 \\
$\mathrm{H}$ & -1.53562700 & 0.30480900 & -1.73923400 \\
$\mathrm{C}$ & -1.61013800 & -1.63542800 & -0.55408500 \\
$\mathrm{H}$ & -2.55761000 & -1.63417100 & -1.09021500 \\
$\mathrm{C}$ & -1.61606500 & -1.94912500 & 0.81104600 \\
$\mathrm{H}$ & -0.67869700 & -2.29882800 & 1.23777800 \\
$\mathrm{C}$ & -2.54674700 & 1.01647900 & -0.05395700 \\
$\mathrm{H}$ & -2.45134200 & 1.53183400 & 0.89515900 \\
$\mathrm{C}$ & -3.83914300 & -1.05060300 & 1.33942100 \\
$\mathrm{C}$ & -2.64773400 & -1.63506200 & 1.70705900 \\
$\mathrm{H}$ & -2.48144600 & -1.77227900 & 2.77324400 \\
$\mathrm{C}$ & -3.74446400 & 1.22363300 & -0.83771100 \\
$\mathrm{C}$ & -5.94645400 & -0.12595400 & 1.86050400 \\
$\mathrm{H}$ & -5.56704400 & -0.02329400 & 2.75201700 \\
$\mathrm{H}$ & -6.4463700 & 0.85505100 & 1.41222400 \\
$\mathrm{O}$ & -4.04477100 & 0.61652100 & -1.86262900 \\
$\mathrm{O}$ & -4.56297100 & 2.19545000 & -0.31353100 \\
$\mathrm{C}$ & -5.72962000 & 2.48176000 & -1.09120100 \\
$\mathrm{H}$ & -6.38093200 & 1.60506800 & -1.16757600 \\
$\mathrm{H}$ & -6.24212300 & 3.29055100 & -0.56708000 \\
$\mathrm{H}$ & -5.45705500 & 2.79692000 & -2.10250000 \\
$\mathrm{H}$ & -4.19160300 & -1.00563300 & 0.31375200 \\
\hline
\end{tabular}

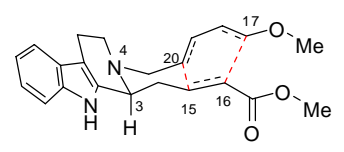

$8 \mathbf{b}:+4.8$

E (B3LYP/6-31G(d)):-1189.074916

E (B3LYP/6-311+G(d,p)//B3LYP/6-31G(d)): -1189.395371 ZPE (B3LYP/6-31G(d)): 0.446201

Imaginary frequencies: 1 $\begin{array}{lll}-4.29865800 & 1.33229000 & -0.19446100\end{array}$ $\begin{array}{lll}-4.29865800 & 1.33229000 & -0.19446100 \\ -5.24347200 & 2.29902500 & -0.54855700\end{array}$ $\begin{array}{lll}-6.50863300 & 1.85656200 & -0.92260700\end{array}$ $\begin{array}{lll}-6.82413200 & 0.48337900 & -0.94520000\end{array}$ $\begin{array}{lll}-5.88130700 & -0.47523200 & -0.59229900\end{array}$ $\begin{array}{lll}-3.39865600 & -0.74478900 & 0.20833500\end{array}$ $\begin{array}{llll}-2.45081400 & 0.21362800 & 0.45450000\end{array}$ $\begin{array}{llll}-5.00125400 & 3.35882500 & -0.53286900\end{array}$ $\begin{array}{llll}-7.26586700 & 2.58391100 & -1.20253400\end{array}$ $\begin{array}{lll}-7.26195800 & 0.17237800 & -1.24283600\end{array}$ $-6.13626000-1.53194100-0.61165100$ $-6.1362600-1.53194100-0.61165100$ $-0.01863100-0.51971600-0.0554300$ $\begin{array}{llll}0.01863100 & 0.51971600 & -0.05541400\end{array}$ $\begin{array}{rrrr}-0.90133100 & 0.52170300 & 1.87891400\end{array}$ $\begin{array}{lll}0.39826400 & -1.80520200 & 1.78856300\end{array}$ $\begin{array}{llll}-0.04740600 & -0.00093900 & -1.01792000\end{array}$ $\begin{array}{llll}-0.21208000 & 1.57560700 & -0.26079600\end{array}$ $\begin{array}{lll}0.37253900 & -2.86799000 & 2.06281500\end{array}$ $\begin{array}{lll}0.53759400 & -1.24946300 & 2.72714900\end{array}$ $-0.91519700-1.45136300-1.26346600$ $-3.09215300-2.20189500-0.38130600$ $-3.09215300-2.20189500 \quad 0.38130600$ $\begin{array}{lll}-3.48389700 & -2.57924900 & 1.33665300\end{array}$ $\begin{array}{lll}-1.23428800 & -2.26920700 & -0.70521300\end{array}$ $\begin{array}{lll}-1.29707500 & -3.40057500 & 0.65601200\end{array}$ $\begin{array}{llll}-3.55171500 & -2.80461100 & -0.41377000\end{array}$ $\begin{array}{lll}-2.98282800 & 1.46987800 & 0.20799000\end{array}$ $\begin{array}{lll}-2.50430200 & 2.34659100 & 0.34409400\end{array}$ $\begin{array}{llll}1.42544400 & 0.43464300 & 0.51440500\end{array}$ $\begin{array}{llll}1.48175600 & 0.65609300 & 1.5825000\end{array}$ $1.6175600-0.65609300-1.58225000$ $1.61042300-1.52093700-0.89470400$ $\begin{array}{lll}1.73362400 & -2.14951200 & -0.3548\end{array}$ $\begin{array}{llll}0.87004100 & -2.67259100 & -0.75642500\end{array}$ $\begin{array}{llll}2.51660900 & 0.95746100 & -0.20439400\end{array}$ $\begin{array}{llll}2.43330700 & 1.22538500 & -1.25166100\end{array}$ $\begin{array}{llll}3.94860300 & -1.25927200 & -0.93558200\end{array}$ $\begin{array}{llll}2.81413700 & -1.98742400 & -1.22974900\end{array}$ $\begin{array}{lll}2.73824300 & -2.37782500 & -2.24218800\end{array}$ $\begin{array}{lll}3.66079200 & 1.43939300 & 0.54131000 \\ 3.94837700 & 1.12754500 & 1.69369900\end{array}$ $\begin{array}{lll}3.649787700 & 1.12754500 & 1.69369900\end{array}$ $\begin{array}{llll}3.94837700 & 1.12754500 & 1.69369900 \\ 4.43686100 & 2.31192600 & -0.18185200\end{array}$ $\begin{array}{rrr}4.43686100 & 2.31192600 & -0.18185200 \\ 5.54174300 & 2.87103700 & 0.53602300\end{array}$ $\begin{array}{lll}5.54174300 & 2.87103700 & 0.53602300 \\ 6.24543100 & 2.09322600 & 0.84964700\end{array}$ $\begin{array}{rrr}6.24543100 & 2.09322600 & 0.84964700 \\ 6.02422300 & 3.56213600 & -0.15748600 \\ 5.19836300 & 3.40423800 & 1.42710600\end{array}$ $\begin{array}{llll}5.19836300 & 3.40423800 & 1.42710600\end{array}$ $\begin{array}{lll}4.23610700 & -0.98003400 & 0.07322300\end{array}$ $4.87292200-1.09988900-1.89808400$ $6.06020000-0.38848800-1.53942200$ $6.75652200-0.52132900-2.36897100$ $5.75652200-0.52132900-2.36897100$ $\begin{array}{rrr}5.83559100 & 0.67254700 & -1.40328100 \\ 6.50083100 & -0.79940100 & -0.62255300\end{array}$

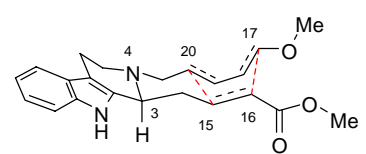

$8 c:+0.1$

E (B3LYP/6-31G(d)): -1189.080582

E (B3LYP/6-311+G(d,p)//B3LYP/6-31G(d)): -1189.402175 ZPE (B3LYP/6-31G(d)): 0.445861

Imaginary frequencies: 1

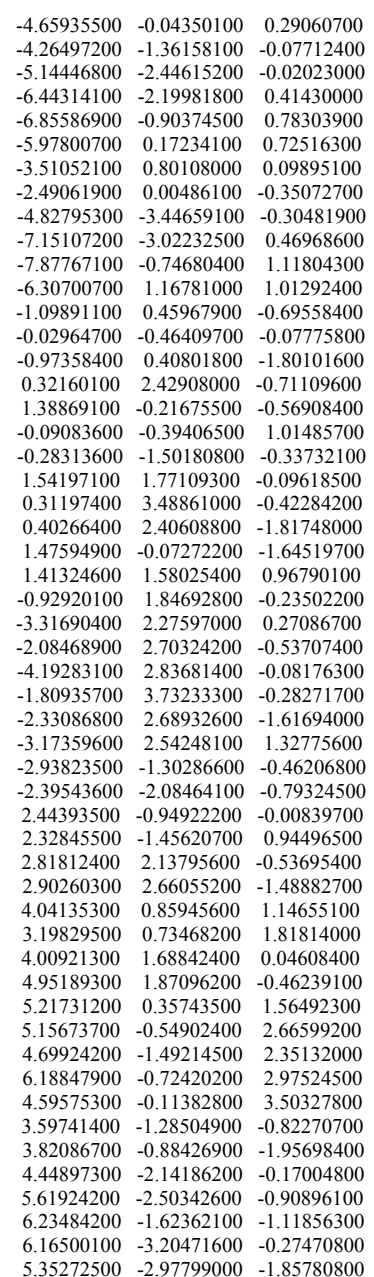




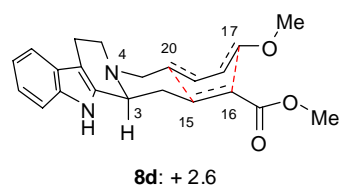

E (B3LYP/6-31G(d)): -1189.077085

E (B3LYP/6-311+G(d,p)//B3LYP/6-31G(d)): -1189.398144 ZPE (B3LYP/6-31G(d)): 0.44586

Imaginary frequencies: 1

\begin{tabular}{|c|c|c|c|}
\hline C & -4.60762600 & 0.05151300 & 0.33834700 \\
\hline $\mathrm{C}$ & -4.38038600 & -1.26484100 & -0.15647100 \\
\hline $\mathrm{C}$ & -5.35393000 & -2.26493600 & -0.08979400 \\
\hline $\mathrm{C}$ & -6.57692200 & -1.93595300 & 0.48678200 \\
\hline $\mathrm{C}$ & -6.82304700 & -0.64172800 & 0.98675500 \\
\hline $\mathrm{C}$ & -5.85205400 & 0.35070100 & 0.91784200 \\
\hline $\mathrm{C}$ & -3.40155900 & 0.80029500 & 0.09865100 \\
\hline $\mathrm{C}$ & -2.51704800 & -0.04655300 & -0.51524000 \\
\hline $\mathrm{H}$ & -5.16506200 & -3.26428300 & -0.47408400 \\
\hline $\mathrm{H}$ & -7.35500200 & -2.69162500 & 0.55252000 \\
\hline $\mathrm{H}$ & -7.78924200 & -0.41880200 & 1.43141400 \\
\hline $\mathrm{H}$ & -6.05397200 & 1.34656100 & 1.30453400 \\
\hline $\mathrm{C}$ & -1.12199600 & 0.30069200 & -0.94569600 \\
\hline $\mathrm{C}$ & -0.06508900 & -0.49186400 & -0.12745600 \\
\hline $\mathrm{H}$ & -0.98716400 & 0.01466500 & -2.00262800 \\
\hline $\mathrm{C}$ & 0.33072400 & 2.22394100 & -1.31100200 \\
\hline $\mathrm{C}$ & 1.36825900 & -0.32302000 & -0.61350200 \\
\hline $\mathrm{H}$ & -0.15022300 & -0.23250300 & 0.93563100 \\
\hline $\mathrm{H}$ & -0.32119700 & -1.55924300 & -0.19387900 \\
\hline $\mathrm{C}$ & 1.51830500 & 1.70838100 & -0.50084300 \\
\hline $\mathrm{H}$ & 0.31273700 & 3.32190500 & -1.28246500 \\
\hline $\mathrm{H}$ & 0.47413900 & 1.94473000 & -2.36547700 \\
\hline $\mathrm{H}$ & 1.48917900 & -0.37314700 & -1.69543500 \\
\hline $\mathrm{H}$ & 1.34910700 & 1.71658400 & 0.57530200 \\
\hline $\mathrm{N}$ & -0.98422000 & 1.76133300 & -0.89225300 \\
\hline $\mathrm{C}$ & -3.04214500 & 2.22620500 & 0.39059700 \\
\hline C & -1.51355300 & 2.38233100 & 0.32650500 \\
\hline $\mathrm{H}$ & -3.50805900 & 2.90390400 & -0.33895700 \\
\hline $\mathrm{H}$ & -1.07253900 & 1.96543500 & 1.25147900 \\
\hline $\mathrm{H}$ & -1.24632300 & 3.44435900 & 0.29870100 \\
\hline $\mathrm{H}$ & -3.39802900 & 2.53482400 & 1.38293700 \\
\hline $\mathrm{N}$ & -3.09309900 & -1.29887900 & -0.66165000 \\
\hline $\mathrm{H}$ & -2.67354600 & -2.08505700 & -1.13327500 \\
\hline C & 2.40625200 & -0.94670400 & 0.09737500 \\
\hline $\mathrm{H}$ & 2.25490500 & -1.29383000 & 1.11508500 \\
\hline $\mathrm{C}$ & 2.81847800 & 1.99680000 & -0.93600900 \\
\hline $\mathrm{H}$ & 2.94947300 & 2.34735200 & -1.95936300 \\
\hline $\mathrm{C}$ & 3.95774500 & 1.01762900 & 0.98964600 \\
\hline $\mathrm{H}$ & 3.09044900 & 1.01316200 & 1.64173300 \\
\hline C & 3.97843100 & 1.65429900 & -0.23397100 \\
\hline $\mathrm{H}$ & 4.94410000 & 1.74982700 & -0.72294900 \\
\hline $\mathrm{O}$ & 5.11363300 & 0.59499000 & 1.53398800 \\
\hline C & 5.00285800 & -0.12359600 & 2.76215000 \\
\hline $\mathrm{H}$ & 4.55477800 & -1.10573400 & 2.58276900 \\
\hline $\mathrm{H}$ & 6.01979000 & -0.24460400 & 3.13900700 \\
\hline $\mathrm{H}$ & 4.40891000 & 0.43843400 & 3.49478700 \\
\hline $\mathrm{C}$ & 3.58185600 & -1.41534700 & -0.61223200 \\
\hline $\mathrm{O}$ & 3.84603300 & -1.20302000 & -1.78808100 \\
\hline $\mathrm{O}$ & 4.40368700 & -2.15892800 & 0.19841700 \\
\hline $\mathrm{C}$ & 5.59473100 & -2.63828200 & -0.43225800 \\
\hline $\mathrm{H}$ & 6.22655700 & -1.80564200 & -0.75568900 \\
\hline $\mathrm{H}$ & 6.11101800 & -3.23347300 & 0.32346100 \\
\hline $\mathrm{H}$ & 5.35592600 & -3.25508600 & -1.30348200 \\
\hline
\end{tabular}

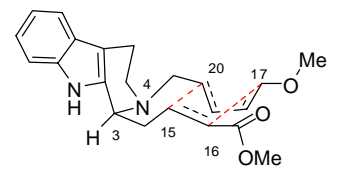

$8 e:+3.0$

E (B3LYP/6-31G(d)): -1189.07800807

E (B3LYP/6-311+G(d,p)//B3LYP/6-31G(d)): 1189.39829972

ZPE (B3LYP/6-31G(d)): 0.446749

Imaginary frequencies: 1

$\begin{array}{lll}0.26956900 & -0.63389200 & 1.58206100\end{array}$ $\begin{array}{llll}1.02201400 & 0.03488000 & 0.44593600\end{array}$ $\begin{array}{lll}1.02201400 & 0.03488000 & 0.44593600 \\ 1.32197100 & -1.50825500 & -0.77528100\end{array}$ $\begin{array}{lll}-0.10139500 & -1.95663300 & -1.05947000\end{array}$ $\begin{array}{lll}-1.00955600 & -1.38976500 & 1.17218300\end{array}$ $\begin{array}{rrr}0.39255100 & 0.51217000 & -0.30616200\end{array}$ $\begin{array}{lll}0.93716000 & -1.32956200 & 2.10161100\end{array}$ $\begin{array}{lll}-0.01051500 & 0.12117900 & 2.33225400\end{array}$ $\begin{array}{lll}-0.64319000 & -1.12906600 & -1.54810600\end{array}$ $\begin{array}{lll}-0.09631700 & -2.79112500 & -1.77383200\end{array}$ $\begin{array}{rrr}-1.33453000 & -1.92985300 & 2.07540000 \\ 1.74473600 & -0.86964300 & -1.54888600\end{array}$ $\begin{array}{lll}1.74473600 & -0.86964300 & -1.54888600\end{array}$ $\begin{array}{lll}1.74473600 & -0.86964300 & -1.54888600 \\ 2.19927700 & 0.74978700 & 0.72716000\end{array}$ $\begin{array}{lll}2.68201500 & 0.69794200 & 1.69679000\end{array}$ $\begin{array}{rrr}2.68201500 & 0.69794200 & 1.69679000 \\ 2.63653000 & 1.76033000 & -0.21100000\end{array}$ $\begin{array}{rrr}2.63653000 & 1.76033000 & -0.21100000 \\ 2.18115900 & -2.37873600 & -0.09123900\end{array}$ $\begin{array}{rrr}2.18115900 & -2.37873600 & -0.09123900 \\ 1.72767600 & -3.24383600 & 0.38766200 \\ 3.52082400 & -2.10461500 & 0.2174100\end{array}$ $\begin{array}{lll}3.52082400 & -2.10461500 & 0.21741100\end{array}$ $\begin{array}{llll}4.05292300 & -2.76448900 & 0.89923400\end{array}$ $\begin{array}{llll}4.19451800 & -0.97728600 & -0.19974200\end{array}$ $\begin{array}{llll}3.84648900 & -0.33649400 & -1.00372100\end{array}$ $\begin{array}{lll}3.84648900 & -0.33649400 & -1.00372100 \\ 2.27707900 & 1.85389300 & -1.38151800\end{array}$ $\begin{array}{rrr}2.27707900 & 1.85389300 & -1.38151800 \\ 3.53830000 & 2.64200100 & 0.33630600\end{array}$ $\begin{array}{rrr}3.53830000 & 2.64200100 & 0.33630600 \\ -0.77280700 & -2.43726900 & 0.15523900\end{array}$ $\begin{array}{rrr}-0.77280700 & -2.43726900 & 0.15523900 \\ -2.15740300 & -0.50762000 & 0.72803200\end{array}$ $\begin{array}{lll}-2.02954200 & -3.13798000 & -0.15067200\end{array}$ $\begin{array}{lll}-2.41574300 & -3.53716700 & 0.79572600\end{array}$ $\begin{array}{lll}-1.79558500 & -3.99576900 & -0.79213600\end{array}$ $\begin{array}{lll}-3.11583500 & -2.25171400 & -0.81202600\end{array}$ $\begin{array}{lll}-2.91952200 & -2.14924100 & -1.88997300\end{array}$ $\begin{array}{llll}-4.08856800 & -2.75658600 & -0.73183000\end{array}$ $\begin{array}{llll}-4.08856800 & -2.75658600 & -0.73183000 \\ -3.13943500 & -0.90493200 & -0.14723700\end{array}$ $\begin{array}{rrr}-3.44193300 & 0.76651600 & 1.19410900\end{array}$ $\begin{array}{lll}-2.44193300 & 0.76651600 & 1.19410900 \\ -3.60442900 & 1.21944400 & 0.59746100\end{array}$ $\begin{array}{lll}-4.27633100 & 2.43731500 & 0.73322800\end{array}$ $\begin{array}{lll}-4.07637800 & 0.18183000 & -0.25617500\end{array}$ $\begin{array}{llll}-5.44659200 & 2.61239600 & 0.00154100\end{array}$ $\begin{array}{lll}-3.90007900 & 3.22067900 & 1.38653900\end{array}$ $\begin{array}{llll}-5.26331600 & 0.38885500 & -0.97991100\end{array}$ $\begin{array}{llll}-5.93595400 & 1.59756300 & -0.84530700\end{array}$ $\begin{array}{llll}-5.99265100 & 3.54806300 & -0.08581900\end{array}$ $-5.64955700-0$. $-5.64955700-0.38593300-1.63758800$ $-6.85385300-1.76692200-1.40189200$ $\begin{array}{llll}-1.84706700 & 1.31664700 & 1.79347600\end{array}$ $\begin{array}{llll}3.95368800 & 3.69602300 & -0.53828000\end{array}$ $\begin{array}{llll}3.09551400 & 4.28404500 & -0.87617300\end{array}$ $\begin{array}{llll}4.63118900 & 4.31763600 & 0.05003200\end{array}$ $\begin{array}{llll}4.46851500 & 3.30042900 & -1.41968900\end{array}$ $\begin{array}{llll}5.44356100 & -0.76636000 & 0.24871500\end{array}$ $6.11056200-0.39727100-0.24782400$ $\begin{array}{llll}5.61438300 & 0.39727100-0.24782400\end{array}$ $\begin{array}{llll}5.61438300 & 1.30068300 & 0.11738000\end{array}$ $\begin{array}{rrr}7.13134700 & 0.34852300 & 0.13436000 \\ 6.13066200 & 0.39725500 & -1.34485900\end{array}$

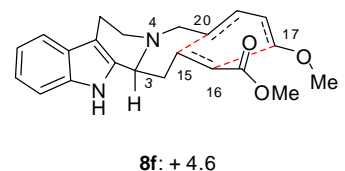

E (B3LYP/6-31G(d)): -1189.07391095

E (B3LYP/6-311+G(d,p)//B3LYP/6-31G(d)): 1189.39523459

ZPE (B3LYP/6-31G(d)): 0.446079

Imaginary frequencies: 1

$\begin{array}{lll}-4.60277500 & -0.09779900 & 0.25646900\end{array}$ $\begin{array}{llll}-4.27010200 & -1.24048600 & -0.52621200\end{array}$ $\begin{array}{lll}-5.19010000 & -2.25921900 & -0.78678300\end{array}$ $\begin{array}{llll}-6.46673700 & -2.12742400 & -0.24877900\end{array}$ $\begin{array}{lll}-6.81846800 & -1.00613400 & 0.52854000\end{array}$ $\begin{array}{lll}-5.90049900 & 0.00601400 & 0.78441900\end{array}$ $\begin{array}{llll}-3.42506700 & 0.72859300 & 0.30563200\end{array}$ $\begin{array}{lll}-2.45222900 & 0.08984300 & -0.41484600\end{array}$ $\begin{array}{llll}-4.92032200 & -3.12511200 & -1.38643200\end{array}$ $\begin{array}{llll}-7.20448300 & -2.90388900 & -0.43207300\end{array}$ $\begin{array}{lll}-7.82413000 & -0.93506200 & 0.93408300\end{array}$ $\begin{array}{llll}-6.18222900 & 0.86573900 & 1.38725100\end{array}$ $\begin{array}{llll}-6.18222900 & 0.86573900 & 1.38725100\end{array}$ $-1.03985700-0.55416400-0.60776100$ $\begin{array}{llll}-0.03274700 & -0.52270600 & -0.10662200\end{array}$ $\begin{array}{lll}-0.87386900 & 0.71659100 & -1.69501400\end{array}$ $\begin{array}{llll}0.34311800 & 2.51967000 & -0.41860000\end{array}$ $\begin{array}{lll}1.30509300 & -0.01214700 & 0.41543700\end{array}$ $\begin{array}{llll}-0.50599700 & -1.06169800 & 0.72378600\end{array}$ $\begin{array}{lll}0.13397200 & -1.25889600 & -0.90569300\end{array}$ $\begin{array}{lll}1.55175500 & 1.64300600 & -0.70605600\end{array}$ $\begin{array}{llll}0.62086900 & 3.30449500 & 0.29559800\end{array}$ $\begin{array}{lll}0.6205891500 & 3.03120600 & -1.36385800\end{array}$ $\begin{array}{lll}0.05891500 & 3.03120600 & -1.36385800 \\ 1.23419300 & 0.54378400 & 1.34540000\end{array}$ $\begin{array}{lll}1.23419300 & 0.54378400-1.34540000\end{array}$ $\begin{array}{lll}1.46925800 & -1.07778000 & -1.63385000\end{array}$ $\begin{array}{lll}-0.81341800 & 1.80457500 & 0.13271300\end{array}$ $\begin{array}{lll}-3.15280400 & 2.05740000 & 0.94030100\end{array}$ $\begin{array}{lll}-1.96825800 & 2.70555500 & 0.21393200\end{array}$ $\begin{array}{lll}-4.02431200 & 2.72127500 & 0.86856500\end{array}$ $\begin{array}{llll}-1.64065100 & 3.60281000 & 0.75204500\end{array}$ $\begin{array}{llll}-2.29531700 & 3.03380100 & -0.79303300\end{array}$ $\begin{array}{lll}-2.91915800 & 1.95370200 & 2.00929800\end{array}$ $\begin{array}{lll}-2.95184500 & -1.10008300 & -0.92180800\end{array}$ $\begin{array}{llll}-2.43997800 & -1.76070500 & -1.48513600\end{array}$ $\begin{array}{lll}2.41423100 & -0.87252700 & 0.33410100\end{array}$ $2.39011500-1.75209400-0.30200600$ $2.82953700 \quad 2.14213700-0.40866900$ $\begin{array}{lll}2.90182300 & 3.00311700 & 0.25326500\end{array}$ $\begin{array}{llll}4.06015700 & 0.25847400 & -1.35782200\end{array}$ $\begin{array}{lll}3.24565400 & -0.11587700 & -1.96949700\end{array}$ $\begin{array}{llll}4.02044100 & 1.47867900 & -0.71341800\end{array}$ $4.95265100-1.84078200-0.28862900$ $-5.23860000-0.38149000-1.48019100$ $5.2386000-0.38149000-1.48019100$ $-18842100-1.6957700-2.04413200$ $4.62628600-1.69281400-2.98755000$ $\begin{array}{llll}6.22211000 & -1.98041300 & -2.24027800\end{array}$ $\begin{array}{lll}4.73665800 & -2.38782400 & -1.33160400\end{array}$ $\begin{array}{lll}3.46937200 & -0.78818600 & 1.32758700\end{array}$ $\begin{array}{lll}3.58570100 & 0.08026500 & 2.18060500\end{array}$ $\begin{array}{lll}4.36528800 & -1.82134600 & 1.20689600\end{array}$ $\begin{array}{llll}5.45209200 & -1.78479300 & 2.13639700\end{array}$ $5.08643000-1.79106400-3.16725100$ $\begin{array}{lll}6.08643000 & -1.79106400 & 3.16725100\end{array}$ $\begin{array}{lll}6.06166300 & -0.88848100 & 1.98699500\end{array}$ 


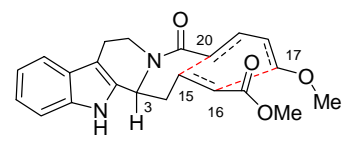

$0.0 \mathrm{kcal} / \mathrm{mol}$

E (B3LYP/6-31G(d)): -1263.12050391 E (B3LYP/6-311+G(d,p)//B3LYP/6-31G(d)): -1263.46397885
ZPE (B3LYP/6-31G(d)): 0.428200

Imaginary frequencies: 1

$\begin{array}{lrrr}\mathrm{C} & 4.67464600 & -0.14691500 & 0.01255500 \\ \mathrm{C} & 4.21605500 & -1.48703500 & 0.15435500 \\ \mathrm{C} & 5.09102200 & -2.57653900 & 0.17590100 \\ \mathrm{C} & 6.45142800 & -2.31056600 & 0.05859800 \\ \mathrm{C} & 6.92878500 & -0.99157200 & -0.07855300 \\ \mathrm{C} & 6.05502400 & 0.08873000 & -0.10270100 \\ \mathrm{C} & 3.50739100 & 0.69531500 & 0.01732700 \\ \mathrm{C} & 2.41943200 & -0.12524600 & 0.15552100 \\ \mathrm{H} & 4.72484300 & -3.59463100 & 0.28190100 \\ \mathrm{H} & 7.15821500 & -3.13564700 & 0.07362200 \\ \mathrm{H} & 7.99811600 & -0.82020800 & -0.16648400 \\ \mathrm{H} & 6.43395500 & 1.10206100 & -0.20853600 \\ \mathrm{C} & 0.99053700 & 0.30671600 & 0.29371300 \\ \mathrm{C} & 0.01169100 & -0.50501800 & -0.60287900 \\ \mathrm{H} & 0.70123900 & 0.11869400 & 1.33811600 \\ \mathrm{C} & -0.33191600 & 2.37442000 & 0.21420800 \\ \mathrm{C} & -1.41971500 & 0.02761700 & -0.69537200 \\ \mathrm{H} & 0.42704400 & -0.54710500 & -1.61730700 \\ \mathrm{H} & -0.00793800 & -1.53722300 & -0.22752800 \\ \mathrm{C} & -1.42839900 & 1.48721600 & 0.72099200 \\ \mathrm{H} & -1.58483600 & 0.72725900 & -1.51049500 \\ \mathrm{H} & -1.15914400 & 0.78848100 & 1.50771800 \\ \mathrm{~N} & 0.89259100 & 1.75482400 & 0.06389600 \\ \mathrm{C} & 3.34165700 & 2.17901400 & -0.10652000 \\ \mathrm{C} & 1.94679700 & 2.47178100 & -0.67022800 \\ \mathrm{~N} & 2.83654900 & -1.44432500 & 0.25254500 \\ \mathrm{H} & 2.23389600 & -2.25033300 & 0.30525300 \\ \mathrm{C} & -2.49329400 & -0.86969300 & -0.50624900 \\ \mathrm{H} & -2.32162000 & -1.84014000 & -0.05001400 \\ \mathrm{C} & -2.73221000 & 2.01678700 & 0.75704000 \\ \mathrm{H} & -2.88148200 & 2.99350300 & 0.30611600 \\ \mathrm{C} & -3.74410200 & -0.06087600 & 1.53173700 \\ \mathrm{H} & -2.83834900 & -0.48491900 & 1.95326300 \\ \mathrm{C} & -3.83769900 & 1.26912200 & 1.13688200 \\ \mathrm{H} & -4.83634300 & 1.66629600 & 0.97738500 \\ \mathrm{O} & -4.87702600 & -0.75024400 & 1.74640400 \\ \mathrm{C} & -4.72800100 & -2.11797800 & 2.12666900 \\ \mathrm{H} & -4.01364400 & -2.22040700 & 2.95393800 \\ \mathrm{H} & -5.71269700 & -2.45300200 & 2.45630900 \\ \mathrm{H} & -4.40363800 & -2.71123500 & 1.26650600 \\ \mathrm{C} & -3.71048000 & -0.70690800 & -1.28826500 \\ \mathrm{O} & -3.99263400 & 0.25463400 & -1.98613800 \\ \mathrm{O} & -4.54297000 & -1.78732900 & -1.15331900 \\ \mathrm{C} & -5.77678700 & -1.68969400 & -1.87344400 \\ \mathrm{H} & -5.59459400 & -1.55769400 & -2.94366300 \\ \mathrm{H} & -6.30117700 & -2.62926300 & -1.69014200 \\ \mathrm{H} & -6.37148700 & -0.84570200 & -1.51210100 \\ \mathrm{O} & -0.52681100 & 3.54356800 & -0.11176300 \\ \mathrm{H} & 1.69226800 & 3.52955500 & -0.60901600 \\ \mathrm{H} & 1.91868900 & 2.18142100 & -1.73086300 \\ \mathrm{H} & 3.46720900 & 2.68051200 & 0.86388500 \\ \mathrm{H} & 4.09187100 & 2.60911600 & -0.78215800 \\ & & & \end{array}$

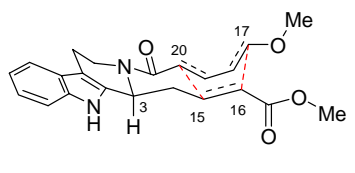

$+0.6$

E (B3LYP/6-31G(d)): -1263.11857263999 E (B3LYP/6-311+G(d,p)//B3LYP/6-31G(d)): -1263.46268937 ZPE (B3LYP/6-31G(d)): 0.427835

Imaginary frequencies: 1

\begin{tabular}{lrrr}
$\mathrm{C}$ & -4.63148800 & -0.17898800 & 0.21722500 \\
$\mathrm{C}$ & -4.21918100 & -1.50138200 & -0.11052300 \\
$\mathrm{C}$ & -5.08082300 & -2.59760800 & -0.01356700 \\
$\mathrm{C}$ & -6.38080400 & -2.35680200 & 0.41901300 \\
$\mathrm{C}$ & -6.81219500 & -1.05556700 & 0.7468500 \\
$\mathrm{C}$ & -5.95182200 & 0.03134500 & 0.65016900 \\
$\mathrm{C}$ & -3.49734800 & 0.67850600 & -0.00528700 \\
$\mathrm{C}$ & -2.46683000 & -0.11846100 & -0.43233200 \\
$\mathrm{H}$ & -4.75028600 & -3.60165700 & -0.26769600 \\
$\mathrm{H}$ & -7.07591100 & -3.18755500 & 0.50429800 \\
$\mathrm{H}$ & -7.83499900 & -0.90366100 & 1.08077800 \\
$\mathrm{H}$ & -6.29489600 & 1.03045700 & 0.90657400 \\
$\mathrm{C}$ & -1.08402700 & 0.34994200 & -0.81062000 \\
$\mathrm{C}$ & 0.00517400 & -0.61863400 & -0.32706000 \\
$\mathrm{H}$ & -1.01077000 & 0.36937400 & -1.91533100 \\
$\mathrm{C}$ & 0.25956400 & 2.37490200 & -0.08683800 \\
$\mathrm{C}$ & 1.41843300 & -0.19334700 & -0.68430600 \\
$\mathrm{H}$ & -0.09482500 & -0.79410600 & 0.75117700 \\
$\mathrm{H}$ & -0.19336200 & -1.58311800 & -0.81556900 \\
$\mathrm{C}$ & 1.39652500 & 1.52874500 & 0.42177800 \\
$\mathrm{H}$ & 1.53114700 & 0.29791000 & -1.64901400 \\
$\mathrm{H}$ & 1.14095100 & 0.94344000 & 1.30133700 \\
$\mathrm{~N}$ & -0.94668800 & 1.74346600 & -0.33870100 \\
$\mathrm{C}$ & -3.33829700 & 2.16475900 & 0.11616900 \\
$\mathrm{C}$ & -2.09648000 & 2.59766400 & -0.67693600 \\
$\mathrm{H}$ & -4.21721200 & 2.69084800 & -0.27995800 \\
$\mathrm{H}$ & -1.80868500 & 3.62564900 & -0.46359700 \\
$\mathrm{H}$ & -2.30131400 & 2.52158900 & -1.75563100 \\
$\mathrm{H}$ & -3.23490800 & 2.47423200 & 1.16604900 \\
$\mathrm{~N}$ & -2.89574200 & -1.43521800 & -0.50656600 \\
$\mathrm{H}$ & -2.33733000 & -2.22333000 & -0.79314500 \\
$\mathrm{C}$ & 2.49328500 & -1.01837600 & -0.33074900 \\
$\mathrm{H}$ & 2.36374100 & -1.83095500 & 0.37793700 \\
$\mathrm{C}$ & 2.68072500 & 2.08832500 & 0.35227200 \\
$\mathrm{H}$ & 2.79894900 & 2.97758700 & -0.25967100 \\
$\mathrm{C}$ & 3.80565000 & 0.28068900 & 1.54772300 \\
$\mathrm{H}$ & 2.91158500 & -0.10868700 & 2.02441800 \\
$\mathrm{C}$ & 3.82791000 & 1.47777900 & 0.85816300 \\
$\mathrm{H}$ & 4.80345300 & 1.87993800 & 0.59909100 \\
$\mathrm{O}$ & 4.96398400 & -0.30407000 & 1.88705400 \\
$\mathrm{C}$ & 4.87022800 & -1.56493600 & 2.55239300 \\
$\mathrm{H}$ & 4.55752000 & -2.33738300 & 1.84338100 \\
$\mathrm{H}$ & 5.87066800 & -1.78827900 & 2.92597400 \\
$\mathrm{H}$ & 4.17019500 & -1.51263800 & 3.39590800 \\
$\mathrm{C}$ & 3.70956100 & -0.99531300 & -1.12548500 \\
$\mathrm{O}$ & 3.96296500 & -0.21014900 & -2.02707900 \\
$\mathrm{O}$ & 4.58176400 & -1.98295200 & -0.74511300 \\
$\mathrm{H}$ & 5.81685500 & -2.00506900 & -1.46884100 \\
$\mathrm{O}$ & 6.37382700 & -1.07625900 & -1.31501600 \\
& 6.37720600 & -2.85347900 & -1.07177900 \\
& 5.63971000 & -2.13407600 & -2.54035400 \\
\hline & 0.38689700 & 3.59184600 & -0.20096700
\end{tabular}

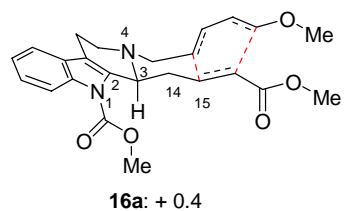

E (B3LYP/6-31G(d)): -1416.95763282 E (B3LYP/6-311+G(d,p)//B3LYP/6-31G(d)): -1417.34130529 ZPE (B3LYP/6-31G(d)): 0.489784 Imaginary frequencies: 1

\begin{tabular}{|c|c|c|c|}
\hline $\mathrm{C}$ & -4.21433300 & -0.86578600 & -0.38000400 \\
\hline C & -4.04774300 & 0.51460000 & -0.63220400 \\
\hline C & -5.03481200 & 1.27187800 & -1.26461800 \\
\hline 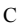 & -6.20115100 & 0.61003000 & -1.64964600 \\
\hline $\mathrm{C}$ & -6.38308300 & -0.76160600 & -1.41011200 \\
\hline $\mathrm{C}$ & -5.39516400 & -1.50760600 & -0.77475300 \\
\hline $\mathrm{C}$ & -3.01904000 & -1.32782800 & 0.28300300 \\
\hline $\mathrm{C}$ & -2.16533800 & -0.27587500 & 0.41544500 \\
\hline $\mathrm{H}$ & -4.89366600 & 2.32702700 & -1.45294000 \\
\hline $\mathrm{H}$ & -6.98295700 & 1.17360100 & -2.15141000 \\
\hline $\mathrm{H}$ & -7.30378500 & -1.24400100 & -1.72691700 \\
\hline $\mathrm{H}$ & -5.53419400 & -2.56933900 & -0.58876600 \\
\hline $\mathrm{C}$ & -0.74246100 & -0.38810100 & 0.91078300 \\
\hline $\mathrm{C}$ & 0.27982000 & 0.13032400 & -0.13209200 \\
\hline $\mathrm{H}$ & -0.62539700 & 0.22906400 & 1.82137100 \\
\hline $\mathrm{C}$ & 0.82144100 & -1.93373600 & 1.93482200 \\
\hline $\mathrm{H}$ & 0.23404900 & -0.50433500 & -1.02342200 \\
\hline $\mathrm{H}$ & -0.01014200 & 1.13915700 & -0.43824100 \\
\hline $\mathrm{H}$ & 0.87651500 & -2.94986600 & 2.34481600 \\
\hline $\mathrm{H}$ & 0.88673200 & -1.24443700 & 2.79905400 \\
\hline $\mathrm{N}$ & -0.44901600 & -1.80289200 & 1.22452900 \\
\hline $\mathrm{C}$ & -2.69384000 & -2.68413200 & 0.82602400 \\
\hline $\mathrm{C}$ & -1.55624400 & -2.53354400 & 1.84023000 \\
\hline $\mathrm{H}$ & -3.57520900 & -3.12307800 & 1.31243100 \\
\hline $\mathrm{H}$ & -1.18324300 & -3.51839800 & 2.14019200 \\
\hline $\mathrm{H}$ & -1.93874900 & -2.03570500 & 2.75237800 \\
\hline $\mathrm{H}$ & -2.38909100 & -3.37566400 & 0.02841500 \\
\hline $\mathrm{N}$ & -2.78280300 & 0.89343000 & -0.11545700 \\
\hline C & 1.69499800 & 0.19199100 & 0.41805400 \\
\hline $\mathrm{H}$ & 1.75343100 & 0.52254600 & 1.45685100 \\
\hline $\mathrm{C}$ & 2.00192000 & -1.69184400 & 1.00345500 \\
\hline $\mathrm{H}$ & 2.92920000 & -1.43192500 & 1.51109200 \\
\hline $\mathrm{C}$ & 2.07628400 & -2.44832200 & -0.17305800 \\
\hline $\mathrm{H}$ & 1.18211000 & -2.99656900 & -0.46147600 \\
\hline $\mathrm{C}$ & 2.74141900 & 0.69323800 & -0.37510600 \\
\hline $\mathrm{H}$ & 2.62904400 & 0.84401000 & -1.44285300 \\
\hline $\mathrm{C}$ & 4.24015200 & -1.60162200 & -0.96603400 \\
\hline $\mathrm{C}$ & 3.10914300 & -2.37264200 & -1.11769900 \\
\hline $\mathrm{H}$ & 2.98811600 & -2.87563500 & -2.07455400 \\
\hline $\mathrm{C}$ & 3.89717100 & 1.25761500 & 0.28514400 \\
\hline $\mathrm{O}$ & 4.21905900 & 1.06951200 & 1.45615300 \\
\hline $\mathrm{O}$ & 4.64821700 & 2.05610000 & -0.54496300 \\
\hline $\mathrm{C}$ & 5.76671300 & 2.69059600 & 0.08180100 \\
\hline $\mathrm{H}$ & 6.48624300 & 1.95295100 & 0.45179600 \\
\hline $\mathrm{H}$ & 6.22414800 & 3.31211000 & -0.69029000 \\
\hline $\mathrm{H}$ & 5.44403500 & 3.30852800 & 0.92476600 \\
\hline $\mathrm{H}$ & 4.55708300 & -1.18391500 & -0.01544900 \\
\hline $\mathrm{O}$ & 5.11529700 & -1.53022300 & -1.98312700 \\
\hline C & 6.28766200 & -0.74132600 & -1.76415400 \\
\hline $\mathrm{H}$ & 6.93131900 & -0.90710600 & -2.62945000 \\
\hline $\mathrm{H}$ & 6.02174700 & 0.31668500 & -1.69029600 \\
\hline $\mathrm{H}$ & 6.80966600 & -1.06141100 & -0.85363500 \\
\hline $\mathrm{C}$ & -2.45300700 & 2.23229600 & 0.09393700 \\
\hline $\mathrm{O}$ & -2.98842800 & 3.16555100 & -0.46698900 \\
\hline $\mathrm{O}$ & -1.47576100 & 2.36811500 & 1.01420100 \\
\hline $\mathrm{C}$ & -1.04934000 & 3.72275600 & 1.24586600 \\
\hline 1 & -0.67260400 & 4.16981100 & 0.32277300 \\
\hline $\mathrm{H}$ & -1.88005100 & 4.32279600 & 1.62427500 \\
\hline $\mathrm{H}$ & -0.25569000 & 3.64875800 & 1.98928900 \\
\hline
\end{tabular}




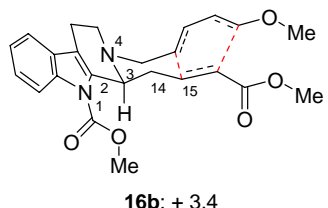

E (B3LYP/6-31G(d)): - 1416.95318247 E (B3LYP/6-311+G(d,p)//B3LYP/6-31G(d)): 1417.33667775

ZPE (B3LYP/6-31G(d)): 0.490043

Imaginary frequencies: 1

\begin{tabular}{|c|c|c|c|}
\hline$c$ & -4.26177100 & -0.90531300 & -0.31186000 \\
\hline $\mathrm{C}$ & -4.17652100 & 0.49752000 & -0.45850800 \\
\hline $\mathrm{C}$ & -5.24285200 & 1.24961200 & -0.95535500 \\
\hline $\mathrm{C}$ & -6.40480200 & 0.56147800 & -1.30589000 \\
\hline $\mathrm{C}$ & -6.50619400 & -0.83212600 & -1.16798600 \\
\hline $\mathrm{C}$ & -5.43888300 & -1.57376400 & -0.67150300 \\
\hline $\mathrm{C}$ & -2.99797600 & -1.36181000 & 0.21341600 \\
\hline $\mathrm{C}$ & -2.18710100 & -0.28160800 & 0.38141400 \\
\hline $\mathrm{H}$ & -5.16636300 & 2.32196500 & -1.06485600 \\
\hline $\mathrm{H}$ & -7.24835900 & 1.12303300 & -1.69797600 \\
\hline $\mathrm{H}$ & -7.42682200 & -1.33428200 & -1.45280400 \\
\hline $\mathrm{H}$ & -5.51470700 & -2.65240200 & -0.56225400 \\
\hline $\mathrm{C}$ & -0.75776800 & -0.36339100 & 0.85603500 \\
\hline $\mathrm{C}$ & 0.27046000 & 0.01018400 & -0.25069500 \\
\hline $\mathrm{H}$ & -0.61312100 & 0.34992500 & 1.67582600 \\
\hline $\mathrm{C}$ & 0.78112900 & -1.84505600 & 2.03964000 \\
\hline $\mathrm{H}$ & 0.25234400 & -0.73506300 & -1.05446100 \\
\hline $\mathrm{H}$ & -0.02473900 & 0.96393300 & -0.70033400 \\
\hline $\mathrm{H}$ & 0.82091500 & -2.83693100 & 2.50919700 \\
\hline $\mathrm{H}$ & 0.84469500 & -1.11378700 & 2.85704400 \\
\hline $\mathrm{N}$ & -0.54097700 & -1.69873900 & 1.43397000 \\
\hline $\mathrm{C}$ & -2.56796600 & -2.75686400 & 0.54995400 \\
\hline $\mathrm{C}$ & -1.03691700 & -2.81030900 & 0.62393600 \\
\hline $\mathrm{H}$ & -2.99775700 & -3.06338700 & 1.51379400 \\
\hline $\mathrm{H}$ & -0.64096700 & -2.81240000 & -0.40772000 \\
\hline $\mathrm{H}$ & -0.71172200 & -3.74426400 & 1.09534500 \\
\hline $\mathrm{H}$ & -2.92885100 & -3.47101300 & -0.20235000 \\
\hline $\mathrm{N}$ & -2.88923700 & 0.89422000 & -0.01608200 \\
\hline $\mathrm{C}$ & 1.67041300 & 0.15864400 & 0.32511400 \\
\hline $\mathrm{H}$ & 1.68523500 & 0.61543700 & 1.31660500 \\
\hline $\mathrm{C}$ & 2.00214700 & -1.64363400 & 1.13306900 \\
\hline $\mathrm{H}$ & 2.87704100 & -1.26833000 & 1.66390500 \\
\hline $\mathrm{C}$ & 2.24064400 & -2.52263300 & 0.06349700 \\
\hline $\mathrm{H}$ & 1.45232000 & -3.21311300 & -0.22275600 \\
\hline $\mathrm{C}$ & 2.73577000 & 0.59923200 & -0.48307100 \\
\hline $\mathrm{H}$ & 2.66196900 & 0.61640500 & -1.56466700 \\
\hline $\mathrm{C}$ & 4.38586500 & -1.56932700 & -0.66549800 \\
\hline $\mathrm{C}$ & 3.33590500 & -2.45386800 & -0.80428300 \\
\hline $\mathrm{H}$ & 3.33873400 & -3.06969500 & -1.70097600 \\
\hline $\mathrm{C}$ & 3.80625900 & 1.34475800 & 0.14619300 \\
\hline $\mathrm{O}$ & 4.07848000 & 1.33650100 & 1.34386500 \\
\hline $\mathrm{O}$ & 4.52905400 & 2.09618000 & -0.74846700 \\
\hline $\mathrm{C}$ & 5.55266500 & 2.90745500 & -0.16343100 \\
\hline $\mathrm{H}$ & 6.30514100 & 2.29379200 & 0.34210900 \\
\hline $\mathrm{H}$ & 6.00390300 & 3.45579600 & -0.99233900 \\
\hline $\mathrm{H}$ & 5.12963600 & 3.60353300 & 0.56669200 \\
\hline $\mathrm{H}$ & 4.61025500 & -1.04526800 & 0.25805000 \\
\hline $\mathrm{O}$ & 5.32663800 & -1.54376100 & -1.62495500 \\
\hline $\mathrm{C}$ & 6.43637300 & -0.66637800 & -1.41783500 \\
\hline $\mathrm{H}$ & 7.16711000 & -0.91337600 & -2.18964700 \\
\hline $\mathrm{H}$ & 6.11727400 & 0.37381300 & -1.52152400 \\
\hline $\mathrm{H}$ & 6.88208200 & -0.82563600 & -0.42802600 \\
\hline $\mathrm{C}$ & -2.54289800 & 2.23405100 & 0.14156700 \\
\hline $\mathrm{O}$ & -1.37931500 & 2.38053100 & 0.80933400 \\
\hline $\mathrm{O}$ & -3.20601200 & 3.16581700 & -0.26522900 \\
\hline $\mathrm{C}$ & -0.95297600 & 3.74338800 & 0.98505400 \\
\hline $\mathrm{H}$ & -0.83024800 & 4.23452400 & 0.01699600 \\
\hline $\mathrm{H}$ & -1.68345500 & 4.29688900 & 1.57972400 \\
\hline
\end{tabular}

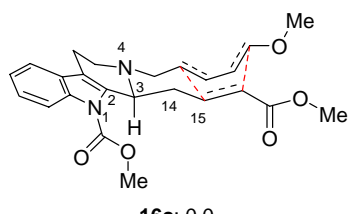

16c: 0.0

E (B3LYP/6-31G(d)): -1416.95703747 E (B3LYP/6-311+G(d,p)//B3LYP/6-31G(d)): 1417.34149748

ZPE (B3LYP/6-31G(d)): 0.489421

Imaginary frequencies: 1

\begin{tabular}{rrr}
4.33363800 & -0.76799500 & 0.54893200 \\
4.12896800 & 0.62919300 & 0.49010900 \\
5.11649500 & 1.53410200 & 0.88308900 \\
6.32187300 & 1.00583300 & 1.34630400 \\
6.54160100 & -0.37940900 & 1.41438700 \\
5.55303400 & -1.27411700 & 1.01671500 \\
3.12861700 & -1.39871600 & 0.06791800 \\
2.23341500 & -0.42636800 & -0.25617500 \\
4.94746100 & 2.60059600 & 0.83365200 \\
7.10540700 & 1.68789600 & 1.66486400 \\
7.49245100 & -0.75437900 & 1.78328500 \\
5.72078400 & -2.34666700 & 1.06828100 \\
0.80365500 & -0.68335600 & -0.66485300 \\
-0.20590900 & -0.02210100 & 0.30310900 \\
0.63487700 & -0.25744200 & -1.67049000 \\
-0.70205500 & -2.44419700 & -1.36689100 \\
-1.61726000 & 0.05053500 & -0.26095400 \\
-0.17971300 & -0.56948200 & 1.25251800 \\
0.11466500 & 1.00145600 & 0.51482900 \\
-1.92180900 & -1.94076800 & -0.61536900 \\
-0.76858100 & -3.53795800 & -1.44223800 \\
-0.71836500 & -2.05585100 & -2.40578800 \\
-1.66267800 & 0.36261100 & -1.30347700 \\
-1.87451500 & -2.18341000 & 0.44478200 \\
0.54805400 & -2.13816800 & -0.68100400 \\
2.82894400 & -2.84955700 & -0.14588500 \\
1.66954300 & -2.95734800 & -1.14047100 \\
3.71164800 & -3.36903500 & -0.54192200 \\
1.32185700 & -3.99406400 & -1.20420400 \\
2.02161200 & -2.66763900 & -2.14980900 \\
2.55722700 & -3.34897200 & 0.79422200 \\
2.82906200 & 0.84875800 & -0.03129600 \\
-2.65435200 & 0.57594600 & 0.52449600 \\
-2.56418600 & 0.63176200 & 1.60533600 \\
-3.17664100 & -1.99273000 & -1.23453000 \\
-3.20970000 & -2.09995000 & -2.31800600 \\
-4.45978800 & -1.37002500 & 0.74771500 \\
-3.67943800 & -1.61206100 & 1.46162100 \\
-4.38262100 & -1.69587000 & -0.59007400 \\
-5.28631300 & -1.57451700 & -1.18106000 \\
-5.63311200 & -0.96150700 & 1.26367500 \\
-5.62002500 & -0.60604400 & 2.64644800 \\
-5.05460200 & 0.31959200 & 2.79083800 \\
-6.66172800 & -0.45097900 & 2.93214000 \\
-5.19307800 & -1.41335600 & 3.25557200 \\
-3.71309500 & 1.34129000 & -0.10752600 \\
-3.88100600 & 1.48698800 & -1.31113800 \\
-4.54207900 & 1.92478900 & 0.81906000 \\
-5.62366100 & 2.67955900 & 0.26575400 \\
-6.28871400 & 2.03576000 & -0.31751300 \\
-6.15790200 & 3.10107200 & 1.11949200 \\
-5.25318300 & 3.47899600 & -0.38247400 \\
2.42093800 & 2.10450500 & -0.48187900 \\
2.96323400 & 3.14804100 & -0.18270600 \\
1.35535400 & 2.01866800 & -1.30349600 \\
0.84110600 & 3.28156600 & -1.76523100 \\
0.57700800 & 3.92014300 & -0.91927600 \\
1.58513900 & 3.79008100 & -2.38292900 \\
-0.04404600 & 3.03226000 & -2.35013500 \\
& & \\
\hline
\end{tabular}

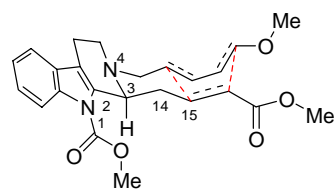

16d: +0.8

E (B3LYP/6-31G(d)): -1416.95623266 E (B3LYP/6-311+G(d,p)//B3LYP/6-31G(d)): 1417.34038914 ZPE (B3LYP/6-31G(d)): 0.48962 Imaginary frequencies: 1 C

\begin{tabular}{rrr}
4.25668600 & -0.95447800 & 0.48171600 \\
4.22633400 & 0.45775700 & 0.43485900 \\
5.31294600 & 1.22912000 & 0.85221400 \\
6.43843500 & 0.55121700 & 1.32146500 \\
6.48520300 & -0.85098700 & 1.37571800 \\
5.39823000 & -1.61208200 & 0.95756300 \\
2.98641800 & -1.43011100 & -0.00995800 \\
2.22611700 & -0.35236600 & -0.34540400 \\
5.27937500 & 2.30849000 & 0.81279300 \\
7.29689200 & 1.12833700 & 1.65409900 \\
7.37894700 & -1.34440700 & 1.74798800 \\
5.43207300 & -2.69767300 & 0.99705500 \\
0.80651400 & -0.45120800 & -0.84465300 \\
-0.21581400 & 0.02363200 & 0.22503700 \\
0.68212000 & 0.19149100 & -1.72266400 \\
-0.73036100 & -2.01196800 & -1.90531700 \\
-1.63409000 & 0.17192800 & -0.31052400 \\
-0.19211500 & -0.65363100 & 1.08814200 \\
0.10606900 & 1.00216000 & 0.59675500 \\
-1.93003100 & -1.72654400 & -1.00450100 \\
-0.78124000 & -3.04837100 & -2.26687100 \\
-0.79473300 & -1.37005100 & -2.79595600 \\
-1.69667200 & 0.65351200 & -1.28592000 \\
-1.84126300 & -2.15951400 & -0.00892200 \\
0.58673700 & -1.83019600 & -1.30980700 \\
2.51389800 & -2.84239800 & -0.17797000 \\
0.98991600 & -2.86511500 & -0.35780200 \\
2.99477200 & -3.29433000 & -1.05684300 \\
0.50892900 & -2.76121900 & 0.63292400 \\
0.67507800 & -3.83392100 & -0.76064100 \\
2.79581300 & -3.45639200 & 0.68791200 \\
2.96436900 & 0.83906000 & -0.08617500 \\
-2.66499800 & 0.54870300 & 0.56548900 \\
-2.54910900 & 0.44624200 & 1.64043200 \\
-3.21329400 & -1.70187800 & -1.56657900 \\
-3.29598400 & -1.61750000 & -2.64982600 \\
-4.40952000 & -1.44445000 & 0.54611500 \\
-3.59389600 & -1.78253700 & 1.17675100 \\
-4.39189900 & -1.54944700 & -0.82954900 \\
-5.32516300 & -1.35400100 & -1.35064900 \\
-5.56588300 & -1.15763400 & 1.17188200 \\
-5.49262700 & -1.01220200 & 2.58978800 \\
-4.95404700 & -0.09472500 & 2.84607100 \\
-6.52257100 & -0.94714000 & 2.94447700 \\
-5.00484300 & -1.88173400 & 3.04953100 \\
-3.75324400 & 1.37521000 & 0.07674700 \\
-3.95490900 & 1.68739400 & -1.08937700 \\
-4.56859200 & 1.79964200 & 1.09657000 \\
-5.67983500 & 2.59977800 & 0.68334600 \\
-6.34439600 & 2.03220700 & 0.02522300 \\
-6.20239100 & 2.87740300 & 1.60082800 \\
-5.34288100 & 3.49507800 & 0.15280800 \\
2.65484000 & 2.16286500 & -0.38989000 \\
3.35870500 & 3.11198000 & -0.11254000 \\
1.47357900 & 2.27028300 & -1.03290300 \\
1.08148300 & 3.61690000 & -1.35637900 \\
1.01775500 & 4.22476900 & -0.45103300 \\
1.80157400 & 4.06776000 & -2.04329500 \\
0.10312700 & 3.52307500 & -1.82751500 \\
& & \\
\hline
\end{tabular}

